\title{
A perspective on 3D surface-related multiple elimination
}

\author{
Bill Dragoset ${ }^{1}$, Eric Verschuur ${ }^{2}$, Ian Moore ${ }^{3}$, and Richard Bisley ${ }^{3}$
}

\begin{abstract}
Surface-related multiple elimination (SRME) is an algorithm that predicts all surface multiples by a convolutional process applied to seismic field data. Only minimal preprocessing is required. Once predicted, the multiples are removed from the data by adaptive subtraction. Unlike other methods of multiple attenuation, SRME does not rely on assumptions or knowledge about the subsurface, nor does it use event properties to discriminate between multiples and primaries. In exchange for this "freedom from the subsurface," SRME requires knowledge of the acquisition wavelet and a dense spatial distribution of sources and receivers. Although a 2D version of SRME sometimes suffices, most field data sets require 3D SRME for accurate multiple prediction. All implementations of 3D SRME face a serious challenge: The sparse spatial distribution of sources and receivers available in typical seismic field data sets does not conform to the algorithmic requirements. There are several approaches to implementing 3D SRME that address the data sparseness problem. Among those approaches are pre-SRME data interpolation, on-the-fly data interpolation, zero-azimuth SRME, and trueazimuth SRME. Field data examples confirm that (1) multiples predicted using true-azimuth 3D SRME are more accurate than those using zero-azimuth 3D SRME and (2) on-thefly interpolation produces excellent results.
\end{abstract}

\section{INTRODUCTION}

During its 75-year history, Geophysics has published many papers about multiple reflections in seismic data. Some of the more interesting papers appear in the January 1948 issue, which was entirely devoted to the question of whether multiple reflections are visible in seismic records. Some authors were firmly convinced that multiples were visible, while others were doubtful. Then there were the cagey ones, such as Dix (1948), who argued in favor of the existence of multiples but conceded, "The evidence... does not yet make their existence 'strictly certain'...."

After improvements in recording technology and more experience with marine seismic data made the existence of multiples obvious, the next question was "How do we get rid of them?" Most of the papers about multiples that have appeared in Geophysics since 1948 address that question. The many intriguing answers fall into three broad categories: exploiting the differences in the properties of primary and multiple reflection events in seismic records, wave-equation-based modeling of multiple reflections using an earth model, and so-called data-driven methods that predict multiple reflections directly from information contained in seismic records. Surface-related multiple elimination (SRME) (Verschuur et al., 1992), the topic of this review paper, falls in the third category. SRME deals only with multiples whose raypaths include one or more downward reflections at the surface. There are data-driven methods related to SRME that predict internal multiple reflections (e.g., Jakubowicz, 1998), but we do not discuss them in this paper.

Because data-driven methods tend to be computationally expensive, early efforts to develop SRME focused on less costly 2D versions of the algorithm. Although sometimes excellent (e.g., Dragoset and Jeričević, 1998; their Figure 3), 2D SRME results were often disappointing because the $2 \mathrm{D}$ algorithm did not encompass the $3 \mathrm{D}$ complexity of geology and data-acquisition geometry. Recent advances in computer hardware have made it feasible to apply advanced versions of 3D SRME to seismic data on a routine basis. Results have been generally outstanding and occasionally astonishing. Figure 1, for example, shows the performance of 3D SRME on a small, full-azimuth data set shot in a circular pattern (Moldoveanu et al., 2008). In spite of the unusual acquisition geometry, 3D SRME has attenuated all surface multiples, including diffracted multiples, exceptionally well. In general, 3D SRME is the most capable of all methods that are widely used for attenuating surface multiples.

Here, we explain in a simple fashion how and why 3D SRME can produce a result such as that in Figure 1. Our paper begins with a discussion of fundamental concepts, explains why a proper application

Manuscript received by the Editor 10 December 2009; published online 14 September 2010.

${ }_{1}^{1}$ WesternGeco, GeoSolutions Technology, Houston, Texas, U.S.A. E-mail: wdragoset@ slb.com.

${ }^{2}$ Delft University of Technology, Delft, The Netherlands. E-mail: d.j.verschuur@tudelft.nl.

${ }^{3}$ WesternGeco, GeoSolutions Technology, Perth, Australia.E-mail:imoore1@ slb.com; rbisley@slb.com.

(C) 2010 Society of Exploration Geophysicists. All rights reserved. 

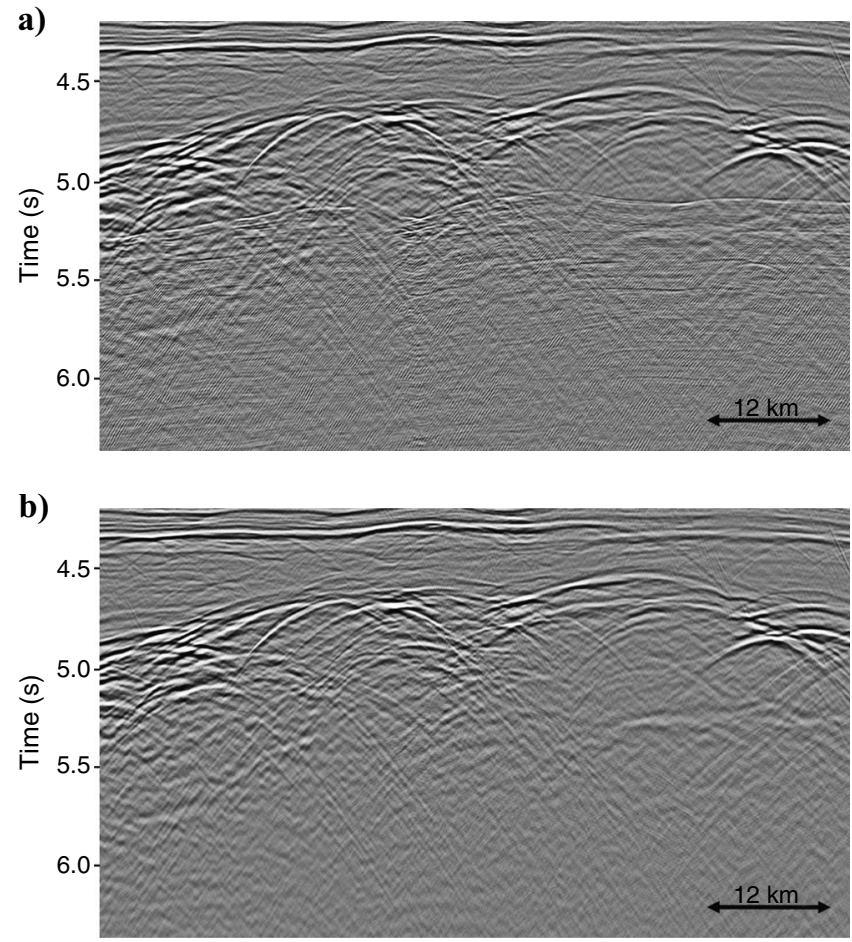

Figure 1. Stack section from a full-azimuth survey shot in a circular pattern. (a) Result without multiple attenuation; (b) result after applying 3D SRME.

Table 1. Meanings of SRME-related terms.

\begin{tabular}{lll} 
Term & Meaning & Remarks \\
\hline Aperture & $\begin{array}{l}\text { The spatial area spanned by } \\
\text { the DRPs in an MCG }\end{array}$ & $\begin{array}{l}\text { The aperture is one dimensional for } \\
\text { 2D SRME and two dimensional } \\
\text { for 3D SRME. } \\
\text { A preconvolution trace adjustment } \\
\text { process used by some SRME } \\
\text { algorithms for on-the-fly interpolation. }\end{array}$ \\
DRP & Differential moveout & $\begin{array}{l}\text { A surface location at which there is a } \\
\text { possibility of a surface multiple } \\
\text { experiencing a downward reflection. } \\
\text { Each trace in an MCG corresponds } \\
\text { to one DRP. }\end{array}$
\end{tabular}

MCG Multiple contribution gather A gather of convolved trace pairs that, when summed, predicts surface multiples for a target trace.

SRME Surface-related multiple elimination

Target A data-set trace for which trace $\quad$ SRME will predict surface multiples

True- $\quad$ An SRME calculation that azimuth honors the actual azimuths SRME of the target traces

Zero- $\quad$ An SRME calculation that azimuth assumes the azimuth of all SRME target traces is the nominal inline azimuth of the survey of SRME must be three dimensional, describes various approaches to 3D SRME, shows some data examples, and ends with a discussion of future directions. For readers unfamiliar with SRME jargon, Table 1 explains some terms that occur frequently in SRME discussions and literature.

\section{SRME FUNDAMENTALS}

\section{Data-driven concepts and history}

Prior to the advent of SRME, attenuation of multiples was often accomplished by using differences in the properties of primary and multiple reflections to discriminate between them. One such property is periodicity. In general, the distribution of reflecting layers within the earth is aperiodic, which means that primary reflections are also aperiodic. On the other hand, some multiples such as shallowwater reverberations can have a periodic structure. That difference is the basis of attenuating multiples by deconvolution methods (Backus, 1959). Another example is stacking velocity-based multiple attenuation. Because the raypaths of a primary reflection and a multiple reflection that have the same zero-offset arrival time traverse different subsurface layers, the two events often have a difference in moveout. If that difference is large enough, simple stacking using the primary reflection's moveout velocity will attenuate the multiple (Mayne, 1962). Radon transform-based multiple attenuation (Hampson, 1986), a popular method, likewise relies on velocity discrimination. All of these approaches as well as other discrimination-based methods share a common trait: they require assumptions about or knowledge of the earth's subsurface.

Another way of attenuating surface multiples is to model them by using the wave equation to propagate a surface-recorded wavefield downward into the subsurface and then back up to the surface. This has the effect of turning primary reflections into first-order multiples, first-order multiples into second-order multiples, and so on. Berryhill and Kim (1986), for example, assume a simple model of the seafloor and then predict water-layer and peg-leg multiples by propagating a surface recording downward to the seafloor and then upward to the surface. Wiggins (1988) describes a more complicated procedure that accounts for a more realistic seafloor. Pica et al. (2005) show that all surface multiples can be predicted using accurate 3D models of the earth's subsurface reflectivity and velocity. In all versions of these modeling procedures, the multiples in the original data are attenuated by adaptively subtracting the modeled multiples.

The data-driven concept for predicting surface multiples is deceptively simple as well as fundamentally different from the wave-equation modeling approach. Figure 2 shows raypaths for a variety of surface multiples. In each case, the raypaths can be divided into two or more segments, each of which could be recorded by a surface seismic experiment. This suggests the possibility that by properly recording and manipulating the appropriate portions of the surface wavefield, any 
surface multiple could be reconstructed directly from a field data set. The trick, of course, is to find an algorithm that automatically carries out that manipulation on a proper set of segments for each surface multiple in a data set. As explained below, SRME accomplishes this in a remarkable fashion: Not only is the manipulation carried out automatically, but it is done so in a way that requires no information other than the recorded traces themselves, the coordinates of their sources and receivers, and the acquisition wavelet. In other words, unlike the modeling methods described above, SRME requires absolutely no knowledge of the subsurface and makes no assumptions about the geologic structure.

One could argue that, in a broad sense, all methods of multiple attenuation are data driven. For example, wave-equation modeling of multiples requires a velocity model calculated from a seismic data set by a procedure such as tomography. Therefore, wave-equation modeling can be classified as a data-driven method because the velocity model is built using the data. However, we strictly define the term data driven as a process whose end result is accomplished by a simple mathematical manipulation of recorded seismic data that requires no intermediate steps, products, interventions, or interpretive analysis. By this definition, SRME is data driven, and the other methods mentioned above are not.

The history of data-driven multiple attenuation began with recognizing there is a convolutional relationship between primary reflections and surface multiples. Watson (1965) writes that relationship as

$$
M_{s 1}(t)=-R_{0} P(t) * r(t),
$$

where $M_{s 1}(t)$ represents first-order surface multiples, $-R_{0}$ is the surface reflection coefficient, $P(t)$ is an upward-traveling sequence of primary reflections that are reflected downward by the surface, the asterisk means convolution, and $r(t)$ is the earth's reflectivity series. Watson explains that one could interpret the quantity $-R_{0} P(t)$ as another surface source of seismic energy. In other words, equation 1 is the familiar convolutional model of the earth, except that the recorded seismogram on the left now consists of surface multiples. Anstey and Newman (1966) also recognize this concept, which they call a retrocorrelogram: "The retro-correlation function...of a seismic trace is obtained by correlating the trace against a time-reversed version of itself. ...During the process..., each primary event on the seismic trace interacts with each primary event on the time-reversed seismic trace to generate a first-order surface multiple event" (p. 411).

Although Watson as well as Anstey and Newman grasp the basic concept of data-driven multiple prediction, their papers indicate the authors do not appreciate the method's full potential. On the other hand, Riley and Claerbout (1976) do. Their key equation, in one dimension and using $Z$-transform notation, is

$$
C(Z)=R(Z)\{1+H(Z) R(Z)\}^{-1} .
$$

Here, $C(Z)$ represents a seismogram that would be recorded if no surface reflections were present, $R(Z)$ is the recorded seismogram that includes surface reflections, and $H(Z)$ is the inverse of the acquisition wavelet.

Equation 2 has two interesting features. First, it indicates that computing $C(Z)$ is clearly an inversion problem. Solving that problem perfectly produces a seismogram which contains no surface multiples. Second, equation 2 introduces the acquisition wavelet into the data-driven concept, a required factor that is explained be- low. Riley and Claerbout solve their equation in two dimensions by using the field data as initial conditions to partial-differential propagation equations. In their solution, they simplify the equations by assuming that the propagating waves travel at angles close to the vertical and that the magnitudes of reflection coefficients are independent of incident angle. Another reason for the narrow angle restriction is their need to avoid converted waves. Thus, although Riley and Claerbout formulate the problem in data-driven terms, they do not solve it in those terms (because of their narrow angle assumption and the use of velocity information for the wavefield propagation).

The data-driven concept for surface multiple prediction reached its full potential in a frequency-space formulation introduced to the seismic industry by the Delphi consortium at Delft University (Verschuur et al., 1992). In fact, Delphi coined the acronym SRME. The Delphi formulation has several important properties: the theory is conceptually simple, extensions from one to two to three dimensions are mathematically straightforward, it is purely data driven, and there are several alternative ways to accomplish the calculation.

Because it is data driven, SRME can predict surface multiples regardless of the details of their propagation through the subsurface. Thus, surface multiples that experience AVO, mode conversions, diffractions, internal reflections, and refractions are all predicted with ease. Furthermore, SRME has no inherent restrictions on wavefield propagation angles or wavefield complexity. Independence from the subsurface, however, does not come without a cost. As we explain later, instead of being burdened by subsurface assumptions, SRME is burdened by strict requirements on data-acquisition characteristics.

During the late 1970s and early 1980s, Royal Dutch Shell developed data-driven SRME independently from the work at Delft University. Perhaps reflecting their surprise that it actually worked, the Shell researchers named it MAGIC. The Shell development remained confidential until early this century (Biersteker, 2001).
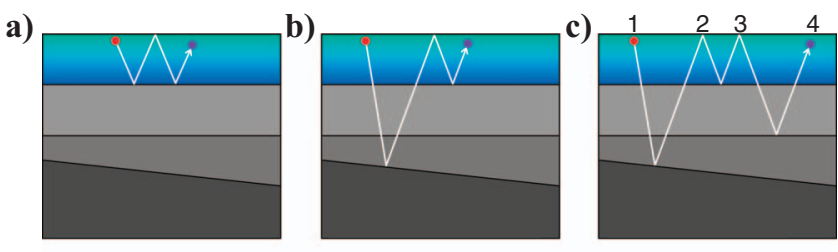

d)

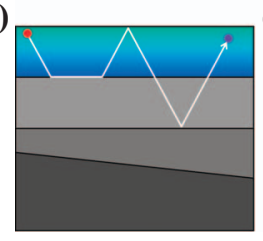

e)
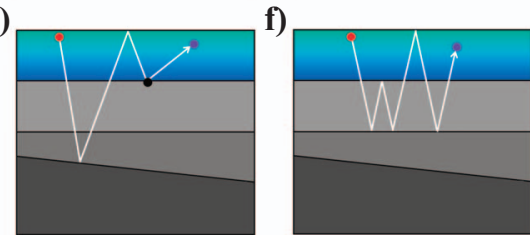

Figure 2. A variety of seismic events represented by their raypaths. (a) Water-bottom multiple. (b) Water-bottom peg leg. (c) Second-order multiple. (d) Refracted multiple. (e) Diffracted multiple. (f) Hybrid multiple. Because each event includes at least one downward reflection at the surface, it is classified as a surface multiple. A key characteristic of all surface multiples is that they can be segmented into subevents which can be recorded in a seismic measurement performed at the surface. For example, the second-order multiple (c) consists of three subevents: 1-2, 2-3, and 3-4. Each subevent is measurable by a surface experiment. Given a suitable set of measurements, SRME can predict all types of surface multiples, including those shown here, without knowing any properties of the subsurface. Red dots are the source, purple dots are the receiver, and the black dot is the diffractor. 


\section{How and why SRME works: The 1D case}

For the workings of SRME, we first consider the simple case of a broadband, horizontal plane wave incident on a horizontally layered earth. We assume the top layer to be acoustic (the marine case). If we define $R(\omega)$ to be the primary reflection response of the earth (without surface-related multiples) and $S(\omega)$ to be the wavelet of this plane wave, then we can write the primary reflection response $P(\omega)$ as

$$
P(\omega)=R(\omega) S(\omega) .
$$

Following equation 1 , we can define the first-order multiples to be the subsurface response of the primaries after downward reflection at the surface with reflection coefficient $r_{0}$ :

$$
M_{1}(\omega)=R(\omega) r_{0} P(\omega)=r_{0} R^{2}(\omega) S(\omega) .
$$

In this way, each order of multiples can be constructed iteratively:

$$
M_{i}(\omega)=R(\omega) r_{0} M_{i-1}(\omega)=r_{0}^{i} R^{i+1}(\omega) S(\omega) .
$$

All surface-related multiples are obtained by summing the results for all orders:

$$
M(\omega)=\sum_{i} M_{i}(\omega)=\left[r_{0} R^{2}(\omega)+r_{0}^{2} R^{3}(\omega)+\ldots\right] S(\omega) .
$$

The total-data value $D(\omega)$ is defined as the sum of the primaries and all surface multiples:

$$
\begin{aligned}
D(\omega) & =P(\omega)+M(\omega) \\
& =\left[R(\omega)+r_{0} R^{2}(\omega)+r_{0}^{2} R^{3}(\omega)+\ldots\right] S(\omega) .
\end{aligned}
$$

An interesting result is obtained when we rewrite the multiples in equation 6 as the total data multiplied by $r_{0} R(\omega)$ :

$$
D(\omega)=P(\omega)+r_{0} R(\omega) D(\omega)
$$

or, using equation 3 ,

$$
D(\omega)=P(\omega)+r_{0} S^{-1}(\omega) P(\omega) D(\omega) .
$$

This describes the relationship between total data and primary data. From this, we can derive equation 2 by writing the primary data explicitly ${ }^{4}$ :

$$
P(\omega)=\frac{D(\omega)}{1+r_{0} S^{-1}(\omega) D(\omega)} .
$$

This direct inversion approach forms the basis of the 2D implementation of Dragoset and MacKay (1993), which involves the inversion of a full matrix per frequency component. Alternatively, the division can be replaced by a series expansion:

$$
P(\omega)=D(\omega)-A(\omega) D^{2}(\omega)+A^{2}(\omega) D^{3}(\omega)-\ldots,
$$

where $A(\omega)=r_{0} S^{-1}(\omega)$. This is the route followed by Verschuur et al. (1992), although extended to the $2 \mathrm{D}$ case, where each term in the series involves matrix multiplications per frequency component. In both approaches, the required input consists only of the measured seismic data, the surface reflectivity, and the source wavelet. The source wavelet is a complication because any discrepancy in the wavelet (in terms of amplitude or phase) will immediately degrade the multiple-elimination result. However, the attractive feature of these expressions is that no other information on the subsurface is required. The series-expansion approach allows precalculation of the prediction terms, such that unknown factor $A(\omega)$ could be estimated by a nonlinear optimization process, assuming that the multiple-free data have minimum energy (Verschuur et al., 1992).

Eventually, workers realized that it is probably better to rewrite equation $8 \mathrm{~b}$ as a recursion process, where the estimated primaries for one iteration serve as the input for the next iteration:

$$
P^{(i+1)}(\omega)=D(\omega)-A(\omega) P^{(i)}(\omega) D(\omega),
$$

where $i$ indicates the iteration number and usually we take $P^{(1)}(\omega)=D(\omega)$. This iterative approach (Berkhout and Verschuur, 1997) has the advantage that, in each iteration, the source signature can be determined by a simple least-squares matching process of the predicted surface multiples to the total data.

Figure 3 shows a simple example of a planewave response that iteratively converges to the primary response using equation 11 . Note that, after one iteration, all multiples are predicted at the correct arrival times but not with the correct amplitudes. More precisely, the higher-order multiples have been overpredicted in amplitude. In practice, this first-order result is often accepted as the predicted multiples, and the adaptive subtraction process - with a time-varying wavelet — takes care of the amplitude discrepancies. Es-

Figure 3. Iterative prediction and subtraction of multiples. (a) Input plane-wave response for a medium with two interfaces. (b-d) Estimated surface multiples after iterations 1, 2, and 5, respectively. (e) True response without surface multiples. (f-h) Estimated primaries after iterations 1,2 , and 5 , respectively.

\footnotetext{
${ }^{4}$ Riley and Claerbout (1976) use $C(Z)$ for primary data $P(\omega), R(Z)$ for total data $D(\omega)$, and $H(Z)$ for the inverse of the acquisition wavelet $S^{-1}(\omega)$.
} 
pecially for deepwater situations, where different orders of multiples are well separated in time, this approach works very well.

Finally, note that if we can assume that the earth is horizontally layered, then equations 3-7, 8a, 8b, and 9-11 remain valid for planewave propagation under nonzero angles. Thus, a CMP gather can be transformed to the frequency-wavenumber or frequency-ray-parameter domain, and each plane-wave component can be treated independently (Kennett, 1979b; Sen et al., 1998).

\section{How and why SRME works: The 2D and 3D cases}

Equations $3-7,8 \mathrm{a}, 8 \mathrm{~b}$, and 9-11 only hold for the strict 1D case (i.e., a horizontally layered earth). In this section, we discuss the multidimensional situation. But let's start with the 1D case and consider the multiple prediction part, with the multiples written as $M(\omega)=R(\omega) r_{0} D(\omega)$ in accordance with equation $8 \mathrm{a}$.

In practice, we do not know the impulse response $R(\omega)$, so we use (an estimate of) the primaries and the wavelet deconvolution filter: $M(\omega)=S^{-1}(\omega) P(\omega) r_{0} D(\omega)$. As stated earlier, the multiples can be interpreted as the surface response of a plane wave, where the total recorded wavefield acts as the source signature. Thus, the recorded wavefield is re-emitted into the subsurface. This concept can be extended to a multidimensional situation. Therefore, we have to consider an exploding surface, where the surface can be thought of as being occupied by a dense grid of sources. Each source emits the surface reflection of the measured upgoing wavefield $r_{0} D\left(x_{r}, y_{r}, \omega ; x_{s}, y_{s}\right)$ at location $\left(x_{r}, y_{r}, z=0\right)$ originating from a seismic source at $\left(x_{s}, y_{s}, z\right.$ $=0)$.

If the subsurface response of all these sources is measured again, we obtain a prediction of the surface-related multiples in a full 3D sense:

$$
M\left(x_{r}, y_{r}, \omega ; x_{s}, y_{s}\right)=r_{0} \sum_{x_{k}, y_{k}} R\left(x_{r}, y_{r}, \omega ; x_{k}, y_{k}\right) D\left(x_{k}, y_{k}, \omega ; x_{s}, y_{s}\right)
$$

where $R\left(x_{r}, y_{r}, \omega ; x_{k}, y_{k}\right)$ describes the primary response of the earth for a source at $\left(x_{k}, y_{k}, z=0\right)$ and a receiver at $\left(x_{r}, y_{r}, z=0\right)$. This is closely related to Huygens' principle (Verschuur, 1992) because we can interpret the surface as a set of secondary sources that emits the upgoing wavefield back into the subsurface such that primaries become first-order multiples, first-order multiples become second-order multiples, etc. Figure 2 shows this pictorially: every multiple reflection consists of a primary reflection combined with another primary or a multiple reflection. Appendix A provides more detail about this intuitive description of SRME.

For the exact quantification of this expression, we must look at wave theory and arrive at the Rayleigh II integral. Usually, the Rayleigh II integral is used to describe one-way wave propagation, where the up/downgoing wavefield at point $A$ is calculated based on the up/downgoing wavefield measured at a surface at $z_{0}$ (see, e.g., Schneider, 1978):

$$
D\left(\vec{r}_{A}, \omega\right)=\frac{1}{2 \pi} \iint_{x, y} \frac{\partial G\left(\vec{r}_{A}, \omega ; \vec{r}_{0}\right)}{\partial z} D\left(\vec{r}_{0}, \omega\right) d x d y
$$

where $G\left(\vec{r}_{A}, \omega ; \vec{r}_{0}\right)$ describes the Green's function (i.e., the impulse response) from a source at $\vec{r}_{0}=\left(x, y, z_{0}\right)$ to point $A$ with $\vec{r}_{A}$ $=\left(x_{A}, y_{A}, z_{A}\right)$. This continuous formulation resembles our more-orless intuitive discrete relation in equation 12 . We can consider the prediction of multiples as a generalized wavefield extrapolation process down and up through the subsurface, described by the impulse responses, $R\left(x_{r}, y_{r}, \omega ; x_{k}, y_{k}\right)$.

In terms of amplitudes, this means the impulse response should contain the vertical derivative term at the source side. In the past, various approaches have been followed to include this effect. Berkhout (1982) and Verschuur et al. (1992) assume that each impulse response of the subsurface has been generated by a dipole source at the surface because a vertical derivative can be simulated by putting two monopole sources with opposite signs spaced closely with one above the other, thus constituting a dipole source. Fokkema and van den Berg (1990) consider an impulse response in terms of the vertical component of the particle velocities (which are in fact vertical derivatives of the pressure wavefield). Weglein et al. (1997) describe the impulse response in terms of monopole sources and receivers, thereby introducing an explicit obliquity factor to introduce the effect of the vertical derivative. As a consequence, their formulation requires deghosting at source and receiver sides of the data, whereas Verschuur and Berkhout's (1997) method allows the source ghost to be present in the data, assuming that a source ghost provides a good estimate of a dipole response.

A physical interpretation of equation 12 can be best obtained when fixing the source and receiver coordinates of the output trace. Then it describes the process of predicting the multiples for one source-receiver combination (i.e., one trace in the seismic data). In the time domain, this reads

$$
m\left(x_{r}, y_{r}, t ; x_{s}, y_{s}\right)=r_{0} \sum_{x_{k}, y_{k}} r\left(x_{r}, y_{r}, t ; x_{k}, y_{k}\right) * d\left(x_{k}, y_{k}, t ; x_{s}, y_{s}\right)
$$

where * means convolution in time. Note that $d\left(x_{k}, y_{k}, t ; x_{s}, y_{s}\right)$ describes a common-source gather and $r\left(x_{r}, y_{r}, t ; x_{k}, y_{k}\right)$ describes a common-receiver gather. These two data gathers are convolved trace by trace, after which the convolution results are summed over the surface coordinates.

Figure 4 visualizes this for one selected multiple event. The interesting fact is that when all convolution events are summed, the physical multiple path (the solid line in Figure 4) will be selected. In terms of traveltimes, the physical path is the one that obeys Fermat's principle of shortest time. In other words, by convolving the traces of a common-source gather and a common-receiver gather, all possible multiple reflection paths at the surface are created and the summation will automatically select the physical contribution. Note that the location of the stationary reflection point at the surface will vary for each type of multiple. Therefore, a preselection of traces to flow into this summation may limit the accuracy of the prediction process.

In Figure 5, this is illustrated for the 2D case in the subsurface model of Figure 4. One shot record is selected from the total data (Figure 5a) and for the common-receiver primary impulse responses the receiver at $x=2100 \mathrm{~m}$ is chosen (Figure $5 b$ ). The result of a trace-by-trace convolution of these two data subsets is shown in Figure $5 \mathrm{c}$, which is often referred to as the multiple contribution gather. The summation of these convolutions is shown in Figure 5d, and this corresponds to the $2 \mathrm{D}$ version of the prediction process (equation $14)$ : 


$$
m\left(x_{r}, t ; x_{s}\right)=r_{0} \sum_{x_{k}} r\left(x_{r}, t ; x_{k}\right) * d\left(x_{k}, t ; x_{s}\right) .
$$

Note that the stationary points in Figure 5c (the apexes of each event) provide the main contributions to the actual multiples in Figure 5d. When this process is repeated for different common-receiver gathers, the predicted multiples for all receiver locations can be obtained (Figure 5e). Subtracting the predicted multiples from the input yields the estimated primaries in Figure 5f. In this case, the perfect result was obtained because we used the true primary-impulse responses in the prediction process. In practice, when starting with the total data as the first estimate of the primaries, the higher-order multiples will be overpredicted and one or more iterations of this process will be required.

\section{Common misconceptions about SRME}

Over the years, several misconceptions about SRME have spread through the geophysical community. A few of them are clarified in the following paragraphs.

\section{Absorption}

The real earth always exhibits absorption of seismic energy, although a larger part of this may be attributed to complex propagation phenomena such as internal scattering within fine layers. A symmetric, first-order multiple will have encountered twice the absorption effect of its corresponding primaries. However, to predict this multiple, the SRME method will convolve two primary responses, such that the total absorption effect in the multiple is predicted properly. Thus, SRME automatically takes absorption effects into account.

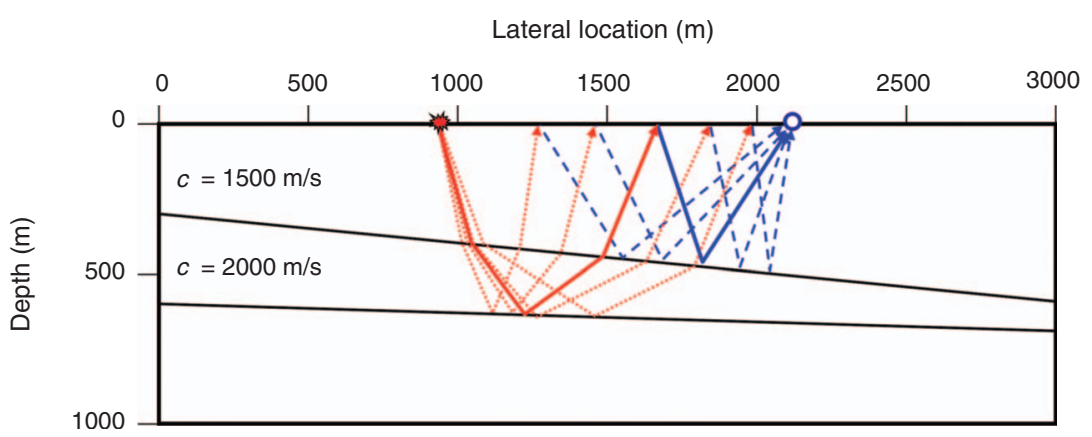

Figure 4. Surface multiples can be predicted by convolving the traces of a common-shot gather (red lines) with those of a common-receiver gather (blue lines). By summing all these convolution results, the physical path (indicated by solid lines) will be obtained and the other contributions will be cancelled.
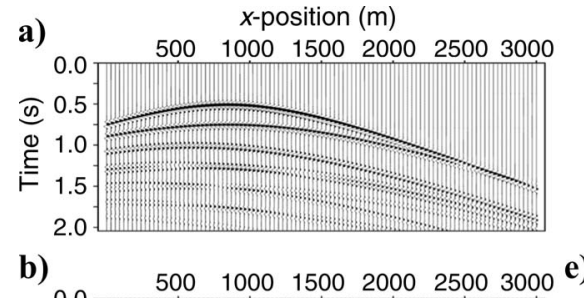

\section{e)}

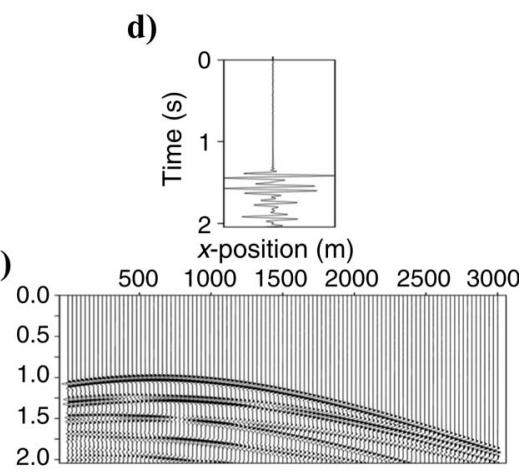

c)
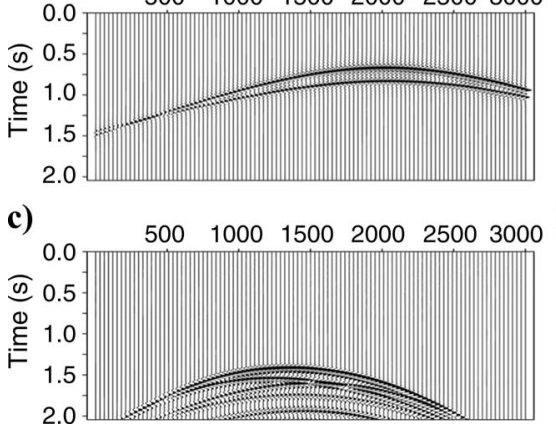

f)

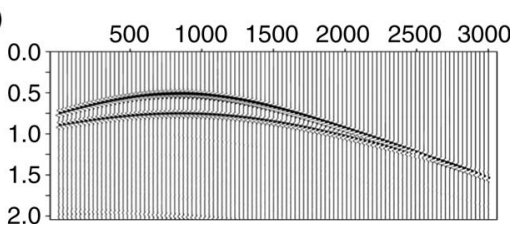

Figure 5. Multiple-prediction mechanism. (a) Shot record with multiples for a source at $x$ $=900 \mathrm{~m}$. (b) Primaries-only impulse response common receiver gather with the receiver at $x=2100 \mathrm{~m}$. (c) Trace-by-trace convolution of (a) and (b), yielding a multiple contribution gather (MCG). (d) Summation of the traces in the MCG, being the predicted surface multiples for a source at $x=900 \mathrm{~m}$ and a receiver at $x=2100 \mathrm{~m}$. (e) Repeating the convolution process for all receiver locations. (f) Primaries, obtained by subtracting the predicted multiples (e) from the input shot record (a).

\section{Wavelet}

A related issue is that some people expect SRME needs the wavelet observed in the seismic data. However, the wavelet that is required for SRME to work properly is the acquisition wavelet, i.e., the wavelet emitted by the source. If this wavelet, because of propagation effects, is modified during its journey through the earth, that modification is part of the impulse response of the earth, not of the wavelet itself. Therefore, estimating a wavelet with SRME and using this wavelet to deconvolve the data will remove the acquisition effect but not the propagation effect.

\section{Internal multiples}

SRME can handle internal multiples in the data. However, from a surface-multiple point of view, these internal multiples are part of the subsurface impulse response and act as primaries. SRME will not remove internal multiples, but it will remove a surface-related multiple of an internal multiple (such as the hybrid multiple in Figure 2f).

\section{Converted waves}

Analogous to the reasoning for internal multiples, converted waves are part of the subsurface impulse responses and are not addressed by SRME. The assumption of SRME is that the measurements are done in an acoustic environment, which is true in the marine case. Any converted wave will reach the receivers as a P-wave traveling through the water layer, and SRME treats them as a regular P-wave primary. Again, surface-related multiples from converted waves will be removed by SRME. If measurements are done in an elastic environment, such as land, things become a bit different. This is described for the 1.5D case by Kennett (1979a, 1979b), implemented in 
an adaptive fashion by Verschuur et al. (1988), and extended to the 2D case by Wapenaar and Berkhout (1989).

\section{Refracted, diffracted, and direct waves}

In general, one can state that any event that is upward traveling from the subsurface and is detected by the receivers can be handled by the SRME method. This includes - besides the previously mentioned internal multiples and converted waves - refracted waves and diffracted waves. All are treated as primary arrivals, from which surface-related multiples associated with these arrivals can be removed (e.g., a surface-related multiple from a refracted wave can be eliminated). The only class of waves that cannot be handled by the SRME method is direct waves (and surface waves in land data). These arrivals must be removed before the SRME process can be carried out.

\section{Data-set requirements}

As described in the iterative implementation of SRME, the measured data themselves are used as the prediction operator (in the first iteration). For the 3D case, we obtain

$$
\hat{m}^{(1)}\left(x_{r}, y_{r}, t ; x_{s}, y_{s}\right)=r_{0} \sum_{x_{k}, y_{k}} d\left(x_{r}, y_{r}, t ; x_{k}, y_{k}\right) * d\left(x_{k}, y_{k}, t ; x_{s}, y_{s}\right),
$$

where ${ }^{\wedge}$ indicates that we will have imperfections because the seismic data do not represent perfect impulse responses of the subsurface. Verschuur and Berkhout (1997) and Dragoset and Jeričević (1998) discuss several of these practical issues. The most important ones follow.

\section{Acquisition wavelet}

The seismic source should behave as a perfect point source, with a unit-valued, broadband zero-phase signature. In practice, the source (array) has directivity effects, a mixed phase, and causal wavelet, and it contains a source ghost. Therefore, these effects should be compensated for in advance or during the prediction and/or subtraction stage. Note that some implementations require that the source ghost be included in order to avoid using the obliquity factor.

\section{Receiver ghost}

The SRME theory assumes that we use the upgoing wavefield at the surface, but in reality we measure the total (pressure) wavefield just below the surface. The main impact of this fact is a receiver ghost that will influence the observed wavelet and - more importantly — create a directivity effect. If not compensated for, multiples will be predicted with a wavelet that varies with propagation angle, which will complicate the subtraction process.

\section{Direct-wave removal}

The direct wave (including its ghost) is not included in the SRME method because it produces no multiples; the data should only contain reflections from the subsurface. Therefore, the direct wave should be removed from the data in advance, usually by a muting process. This step becomes more complicated when the water layer is shallow and the direct wave interferes with the early reflection event(s).

\section{Missing near-offset reconstruction}

The summation in equation 15 or 16 should cover all downward reflection points (DRPs) at the surface where multiples have bounced. Therefore, the aperture of the data should be large enough to capture all of these locations. For the far offsets, this is usually not a problem. However, at small offsets - where the nearest offset typically is $100-200 \mathrm{~m}$ - this produces severe edge effects in the predicted multiples, especially in shallow-water situations (see, e.g., Verschuur, 1991, 2006; Dragoset, 1993). Therefore, a very important preprocessing step is to extend the offsets to zero offset (see, e.g., Kabir and Verschuur, 1995) and even beyond by applying reciprocity.

\section{Adaptive subtraction}

We have explained how SRME predicts multiples. If the process were perfect, that would be the end of the story; the predictions could be subtracted from the original data to remove the surface multiples. Unfortunately, for many reasons, such predictions are never perfect. First, if SRME prediction consists of just the first-order iterative term, then, as explained, the amplitudes of all multiples beyond first order are incorrect. Second, if SRME prediction uses an acquisition wavelet that contains errors or no acquisition wavelet at all, then the accuracy of the predictions will be lowered. Third, errors in predicted multiples can occur for other reasons, including shot-to-shot variations in the source signature, imperfect calibration of the receivers, and errors that occur when a field data set imperfectly matches the sampling requirements of the SRME algorithm. Finally and most importantly, if multiples are predicted by 2D SRME, they can grossly mismatch actual multiples because of the limitations of the $2 \mathrm{D}$ assumption. Because of these phenomena, simple subtraction of predicted multiples from the original seismic traces generally gives poor results. Note, too, that poor subtraction results can be compounded when using an iterative SRME calculation that includes a step-by-step subtraction.

When they are not too large, errors in predicted multiples often can be handled by adaptively subtracting the predictions from the original data. The most commonly used form of adaptive subtraction is least-squares matched filtering (Abma et al., 2005). Within a data window, a filter is designed that, when applied to the multiple predictions, will minimize the energy in the window after the filtered predictions are subtracted. The two main assumptions in this procedure are that within the data window the predictions do not correlate with primary reflections and that a single filter exists that can accomplish the matching. The validity of these assumptions depends on the size of the window, the filter length, the amount of variation in the predicted multiple errors, and the geologic distribution and character of primaries and multiples.

Because of the known limitations of least-squares adaptive subtraction (van Borselen et al., 2003), much effort has been expended to find alternative methods of adaptive subtraction that can complement least-squares adaptive subtraction or replace it. Discussion of these techniques is beyond the scope of this review. However, adaptive subtraction is an active research area and further developments are likely.

Regardless of the type of adaptive subtraction that one uses, wide experience has revealed the following:

- In general, a more accurate prediction of multiples reduces the challenge of obtaining a good adaptive subtraction. 
- The errors in multiples predicted by 2D SRME can be so great (Dragoset et al., 2006; their Figure 9) that no adaptive subtraction algorithm has a chance of overcoming them. The only way around this problem is to predict the multiples with $3 \mathrm{D}$ rather than 2D SRME.

- Adaptive subtraction is still necessary with 3D predictions because of the reasons mentioned earlier. However, because of improved prediction accuracy, the errors in predictions from $3 \mathrm{D}$ SRME generally are within the bounds of what can be handled by adaptive subtraction algorithms.

\section{D SRME}

\section{Why 3D SRME is necessary}

Fundamental SRME theory is naturally three dimensional, in that it accommodates a fully 3D earth. Until fairly recently (e.g., Kleemeyer et al., 2003), however, SRME has almost invariably been implemented in a $2 \mathrm{D}$ sense.

Implementations that are two dimensional assume that the geology and acquisition geometry are two dimensional, with the only concession to the true 3D nature of the problem's being some accommodation of the approximately pointlike nature of the source. Historically, 2D implementations have been favored because typical acquisition geometries provide insufficient sampling in at least one direction for 3D SRME (see following section). In addition, the cost (CPU requirement) for 3D algorithms was prohibitive.

Figure 6 illustrates the limitations of a 2D algorithm when crossline dip or cable feather is present. Figure 6 a illustrates the effect of crossline dip on multiples predicted with a 2D algorithm. The red raypath indicates the multiple recorded on the $(S, R)$ trace (source $S$, receiver $R$ ) for a reflector with crossline dip. The DRP for this multiple, $X$, is on the shallow (updip) side of the 2D line; $X$ has nonzero crossline offset $y$ and is therefore outside the 2D aperture, which lies along the shot-receiver line only. The true traveltime for the multiple is equal to the sum of the primary traveltimes on traces $(S, X)$ and $(X, R)$, but neither of these traces is in the $2 \mathrm{D}$ data set. The traveltime predicted by a $2 \mathrm{D}$ algorithm corresponds to $X^{\prime}$, the point on the $2 \mathrm{D}$ line that minimizes the sum of the primary traveltimes on $\left(S, X^{\prime}\right)$ and $\left(X^{\prime}, R\right)$. This will always be greater than the true multiple traveltime when the DRP is not on the $2 \mathrm{D}$ line.

Figure $6 \mathrm{~b}$ illustrates the effect of cable feather for the case in which feather aligns the traces with the dip direction. The traveltime for the multiple on trace $(S, R)$, indicated by the red raypath, is equal to the sum of the traveltimes on traces $(S, X)$ and $(X, R)$. Although the a)

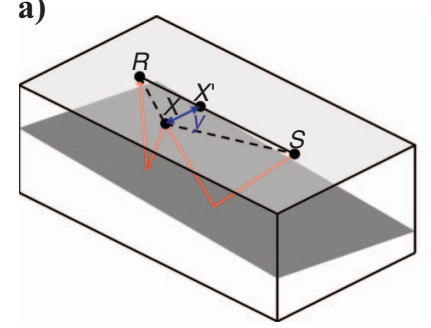

b)

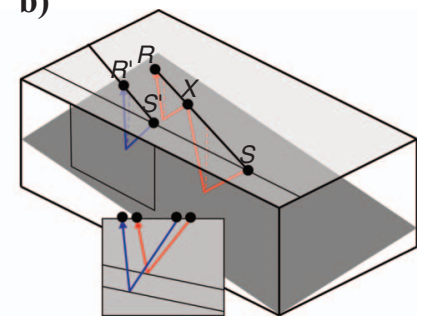

Figure 6. Limitations of 2D SRME when (a) crossline dip or (b) feathering is present. The effect of cable feather is shown for the special case when feather aligns the traces with the dip direction. The inset is a side view of reflection raypaths for reflections $(X, R)$ and $\left(S^{\prime}, R^{\prime}\right)$. Refer to the text for a detailed explanation of this figure. data set will contain a trace close to $(S, X)$, it will not contain a trace close to $(X, R)$; the nearest trace, $\left(S^{\prime}, R^{\prime}\right)$, must be used instead. The primary traveltimes for $(X, R)$ and $\left(S^{\prime}, R^{\prime}\right)$ are not the same (see the inset), and this will lead to errors in the predicted traveltime, which could be of either sign. In general, the DRP will not lie along the cable. Neither the trace $(S, X)$ nor the trace $(X, R)$ will be in the recorded data set, leading to errors in the predicted multiples, which are a complex function of the geology and the acquisition geometry.

Although efforts are often made to accommodate the assumptions of the $2 \mathrm{D}$ algorithm - for example, by shooting in the direction of maximum structural dip and by keeping cable feather to a minimum — violations of the $2 \mathrm{D}$ assumptions inevitably occur in practice. Wide-azimuth (WAZ) and orthogonal geometries are also inherently incompatible with 2D algorithms.

Violations of the 2D assumptions lead to errors in the predicted multiples (van Dedem and Verschuur, 1997; Dragoset and Jeričević, 1998; Nekut, 1998; Ross et al., 1999; Dragoset et al., 2006). The timing component of these errors is predictable, given a model of the geology, and is also measurable in the data, e.g., using crosscorrelations. Figure 7 illustrates that the errors vary rapidly and can have magnitudes on the order of $100 \mathrm{~ms}$. Moreover, the timing errors vary according to the mode of multiple in a complex manner. Unless these $3 \mathrm{D}$ effects are small and slowly varying, adaptive subtraction is unable to compensate for them, and the $2 \mathrm{D}$ algorithm is ineffective.

The 3D nature of the acquisition geometry can be addressed to some degree by extending the $2 \mathrm{D}$ algorithm to a $2.5 \mathrm{D}$ algorithm (Matson and Corrigan, 2000). However, such an extension does not address $3 \mathrm{D}$ geologic effects and often suffers from issues relating to inadequate sampling in the crossline direction.

The motivation for a 3D algorithm is clear. Given suitable data, a
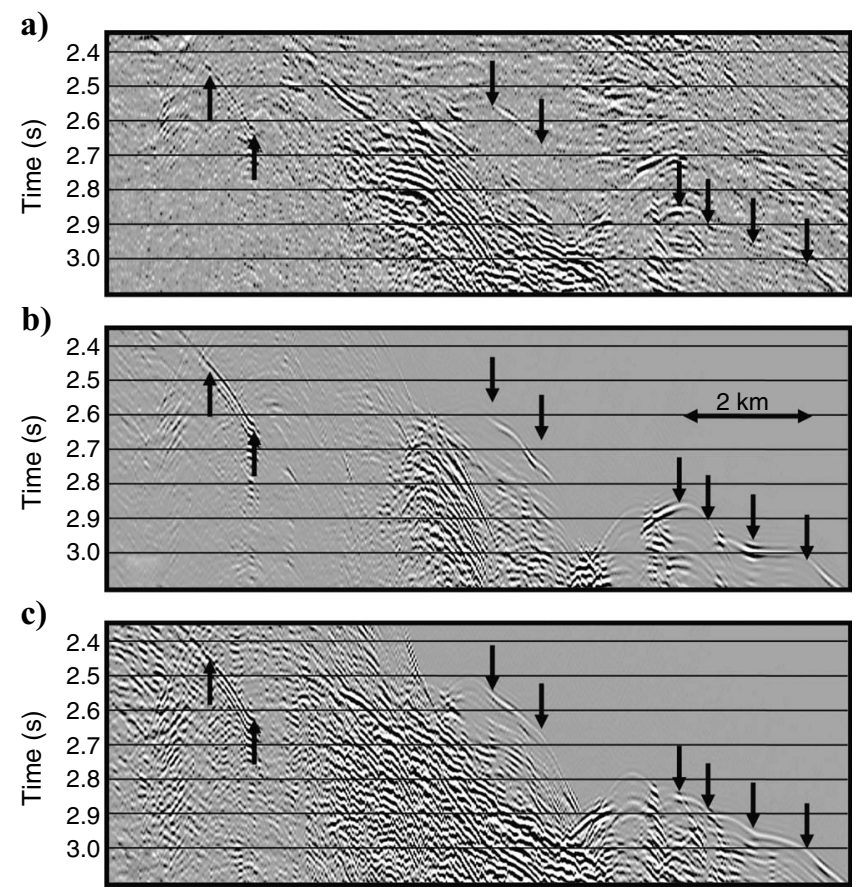

Figure 7. The 200-m offset plane for a field data set at the level of the first-arrival multiple (arrows) for (a) the input data, (b) 2D predicted multiples, and (c) 3D predicted multiples. It is clear that the 3D prediction is much more accurate than the $2 \mathrm{D}$ prediction. The timing errors for the 2D prediction are about $80 \mathrm{~ms}$ at the center of the line. (from Moore and Bisley, 2005) 
3D algorithm will theoretically avoid all of the assumptions discussed here. Even taking into account practical issues such as noise and imperfect sampling, we can expect a model that is significantly more accurate than that produced by a $2 \mathrm{D}$ algorithm. Advances in computer power have greatly reduced the impact of this as a barrier to the implementation of 3D algorithms, leaving data-sampling issues as the main challenge to overcome.

\section{Data-sampling requirements for 3D SRME}

At the heart of any SRME algorithm is the integration over the aperture of convolutions of pairs of traces (see SRME Fundamentals). This integration is typically performed by stacking (summing) the convolutions that form the MCG. The sampling density in the MCG should therefore be sufficient to avoid having aliased energy contaminate the stack trace. This condition in turn determines the desired sampling of the recorded data.

The aliasing condition for the MCG depends on the maximum frequency of interest and the maximum dip of events in the MCG. The actual shape of events in the MCG is complex in general, but a useful indication of the maximum dip can be obtained by considering the asymptotic behavior of events as the DRP moves to infinity. In that case, the traveltime in the MCG is essentially twice the traveltime from the source or receiver to the DRP, and hence the apparent velocity is half of the physical velocity. Because only the stack trace of the MCG is required, aliasing only becomes a problem when the aliased energy contaminates the zero-wavenumber component of the spectrum. Therefore, the sampling requirement for the MCG is equivalent to that for unaliased shot and receiver gathers. An example of this phenomenon is shown for 2D SRME by Dragoset et al. (2006; their Figures $4 b$ and $5 b$ ). That is, a filter sufficient to remove aliasing from input shot records (Figure 4b) prevents aliasing artifacts from appearing in predicted multiples (Figure 5b).

In practice, the maximum observed dip in the MCG is generally less than the asymptotic limit because the aperture is limited. The required sampling within the MCG can be determined by testing on sparse but representative target traces.

The aperture needs to include the DRPs for any multiples of interest. Because of the integration process, the word "include" should be taken to mean inclusion of a Fresnel zone around the apex in the MCG, such that the correct constructive interference is achieved by the stacking process. In addition, it is normal to taper amplitudes at the edge of the MCG to avoid edge effects. The size of this taper should also be taken into account when determining the size of the aperture.

To predict multiples for a given target trace, theoretically we need to record areal shot and receiver gathers with sampling and apertures matching those of the MCG (Dragoset and Jeričević, 1998). Typically, this means we require inline and crossline shot and receiver spacings on the order of tens of meters and inline and crossline offsets on the order of several kilometers. These requirements are not achievable in practice, and some form of interpolation is required. Before considering this practical issue, though, we consider the implementation of 3D SRME given ideal data.

\section{D SRME calculation given ideal data}

Given ideal data, the 3D SRME algorithm is very simple. The following procedure illustrates the basic steps of the algorithm.

A target trace $(S, R)$ is a single source-receiver pair from a dense regular survey grid, for which we generate a multiple model (Figure 8a). Figure $8 \mathrm{~b}$ shows an MCG aperture, which is designed to include the DRPs of interest. The MCG is formed from complementary gathers, an areal source gather with its source at $S$ (Figure 8c), and an areal receiver gather with its receiver at $R$ (Figure $8 \mathrm{~d}$ ). In an iterative implementation, one of these gathers is composed of estimated primary traces; the other is composed of data traces. A single contribution to the MCG corresponding, for example, to DRP $X$ is created by convolving a trace with a source at $S$ and a receiver at $X$, with a second trace with a source at $X$ and a receiver at $R$. The convolution step is repeated for every DRP in the aperture and the collection of convolutions is stacked.

This description omits some of the details, such as the obliquity factor, effect of the ghosts, and the source signature. These effects can be accounted for if necessary in practice but are of secondary importance to the issues created by the fact that sampling of field data is usually far from ideal.

The 3D SRME process is demonstrated in Figure 9, where we consider a synthetic data set for a model that consists of two diffractors in a homogeneous medium. We consider the data measured along the inline direction for $y=0$ and show the inline zero-offset traces in Figure 9a. The crossline locations of the diffractors are $y=$ $-300 \mathrm{~m}$ and $y=+200 \mathrm{~m}$, i.e., they are out of plane. If we assume that we only have measurements at $y=0$, the $2 \mathrm{D}$ situation, then the predicted multiples are given in Figure 9b. Note that the locations of these predicted multiples do not coincide with the true multiples. Especially for the left diffractor (being the more out of plane), this difference is quite large (about $150 \mathrm{~ms}$ ). Because the error is different for each multiple event, adaptive subtraction cannot overcome the problem. This is visible in the 2D multiple-removal result in Figure $9 \mathrm{e}$. The multiples for the second diffractor could be reasonably well attenuated, but the multiples for the first diffractor could not be addressed.

Next, the full 3D prediction is carried out, assuming a dense areal sampling in sources and receivers (Figure 9c). Note that all multiples are predicted with the correct kinematics. Figure $9 \mathrm{~d}$ shows the MCG a)

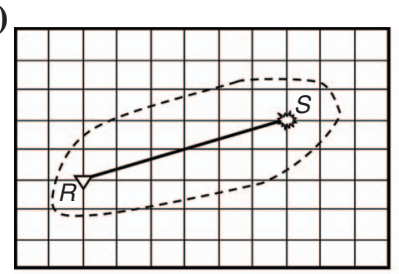

b)

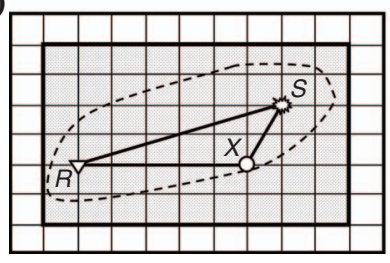

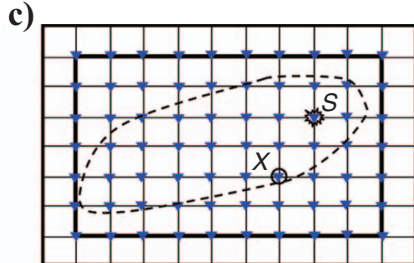

d)

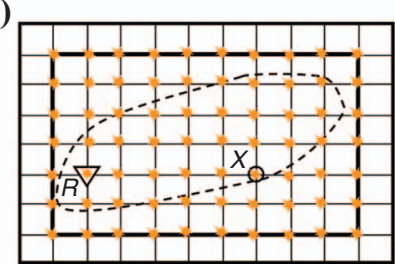

Figure 8. Plan view of 3D SRME, illustrating the MCG and required traces. (a) A target trace. The dashed line indicates the region that includes all downward reflection points for the multiples of interest. (b) The MCG aperture for the target trace (shaded box) and an example DRP, $X$. (c) The required areal shot gather, with the source at $S$. Receivers are required at all grid nodes (blue triangles) included in the aperture. Trace $(S, X)$ required for the example DRP is annotated. (d) The areal receiver gather, with the receiver at $R$. Sources are required at all grid nodes (yellow stars) included in the aperture. Trace $(X, R)$ required for the example DRP is annotated. 
for the middle trace in Figure 9a (indicated with the dashed line) after the inline summation. This means that in equation 16, the summation over $x_{k}$ has been carried out, providing a prediction result for each crossline location $y_{k}$. After summation in the crossline direction, the energy at the apexes will stack into the predicted multiples. The lateral location of each apex defines the DRP of the corresponding multiple. Indeed, the timing of the apex locations in Figure 9d corresponds with the true multiples in Figure 9a, as indicated by the times at which the dashed line intersects them. Note that the 2D multiple prediction for this output trace is obtained by selecting the trace at $y=0$ in Figure 9d, which is quite wrong for two of the events. As expected, the subtraction of the $3 \mathrm{D}$ predicted multiples is close to perfect, as visible in Figure $9 \mathrm{f}$.

For this synthetic example, we were able to use the true primaries as the multiple prediction operator to get first- and second-order multiples in the correct amplitude balance. In practice, the primaries are not available, and we need the iterative implementation of SRME (equation 11). In the second and later iterations of an iterative implementation, the source-side or the receiver-side trace will contain estimated primaries rather than data. Although it does not matter whether the primary estimate is used on the source or receiver side, the choice must be implemented consistently for all convolutions.

\section{Application of 3D SRME to field data}

Field data are far from ideal in many respects. The most important shortcoming in practice is inadequate sampling, in terms of sampling density and range of offsets acquired. Many workers (e.g., Lin et al., 2005; Bisley and MacNeill, 2008; McHugo et al., 2009) show the detrimental effects of insufficient aperture and sampling on multiple models using field data.

All acquisition geometries used today exhibit nominal sampling densities that are too coarse in at least one direction. For marine- a)

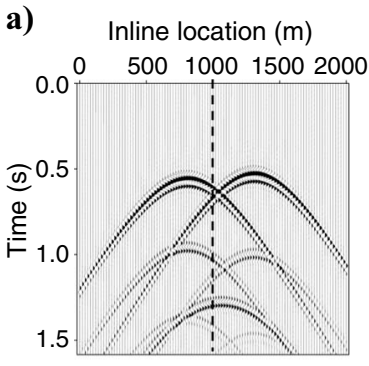

d)

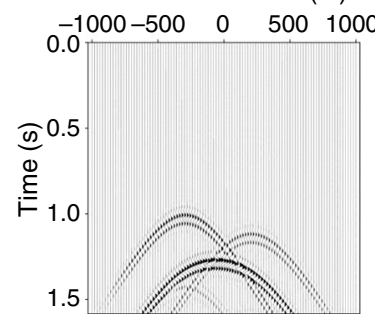

b)

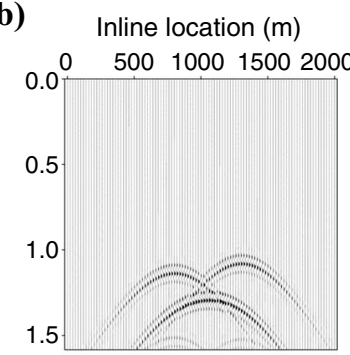

e)

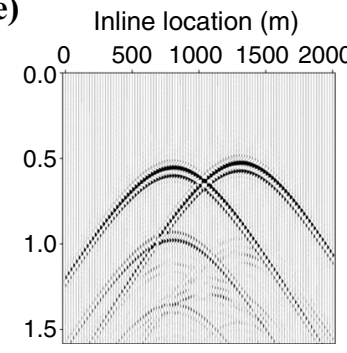

c) Inline location $(\mathrm{m})$

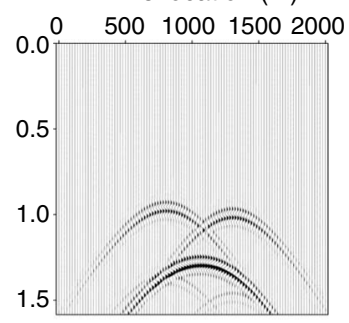

f)

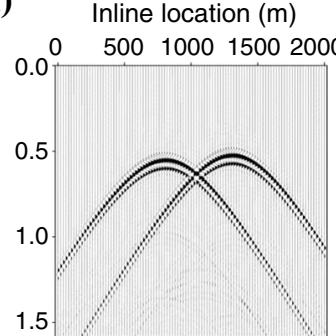

Figure 9. Illustration of $2 \mathrm{D}$ versus $3 \mathrm{D}$ multiple prediction for a model with two diffractors in a homogeneous medium. The diffractors are located at $-300 \mathrm{~m}$ and $+200 \mathrm{~m}$ out of the recording plane. (a) Inline zero-offset section of the model. (b) The 2D predicted multiples along the inline direction for the zero-offset locations in (a). (c) The 3D predicted multiples, using a dense inline and crossline distribution of sources and receivers. (d) Multiple contribution gather after inline summation for the middle trace in (a), indicated by the dashed line. Note that the apex locations in the MCG coincide with the times at which the dashed line in (a) intersects the multiples. (e) The $2 \mathrm{D}$ subtraction result. (f) The $3 \mathrm{D}$ subtraction result. streamer data, the worst-sampled direction is typically the crossline source direction, which is often undersampled by a factor of 10 or more compared to the desired sampling. This coarse sampling is often exacerbated by coverage holes that occur as a result of obstrucdoes not fit the required sampling of the MCG.

Typical geometries also do not record areal shot and receiver gathers with sufficient extent to cover the desired aperture. For marine in particular, the maximum crossline offset is often insufficien lation and extrapolation before they are suitable for 3D SRME.

Many other characteristics of field data violate the assumptions of the 3D SRME algorithm to a lesser extent. For example, the tidal state and/or water velocity vary throughout the acquisition, such that recorded data are inconsistent with a single-earth model. Field propriate preprocessing. Some of the implications of these practical issues are described in more detail by Dragoset et al. (2006).

\section{IMPLEMENTATIONS OF 3D SRME}

Over the years, various implementations of 3D SRME have been reported in the literature, their difference mainly found in the way

\section{Data interpolation/extrapolation/regularization}

In the early days of 3D SRME (the late 1990s and early 2000s), the general concept was to interpolate and extrapolate all data required to carry out the $3 \mathrm{D}$ SRME process. Thus, the difference between the presented methodologies was determined by the type of interpolation method used. The first reported implementation of 3D SRME on field data was by Biersteker (2001), later expanded on by Kleemeyer et al. (2003). Although they do not describe the exact interpolation method, it is clear that they interpolate the data before doing the summations. Along similar lines, Lin et al. (2004) and Matson et al. (2004) advocate to first interpolate the data to a dense grid, followed by full 3D prediction.

Baumstein and Hadidi (2006) are clearer about their interpolation methodology: they use dip moveout (DMO) and normal moveout (NMO) to project the data onto a dense, regular grid, a process they call inverse shot record DMO (ISRDMO).

\section{Crossline reconstruction of multiple contributions}

To solve the 3D SRME problem, van Dedem and Verschuur $(2000,2005)$ postpone the interpolation step to a later stage. First, the inline summation is carried out, this being the summation 
over $x_{k}$ in equation 16 . This summation can usually be done without problem because in traditional narrow-azimuth 3D marine data acquisition, the inline coordinates are well sampled in terms of sources and receivers. This results in the so-called crossline multiple contribution gathers (MCGs) where only a few contributions are present, one for each contributing source line. For a typical 3D marine data set with dual sources, this gives four contributions per output trace. Such an MCG consists of curved events, where the apex of each event represents the exact multiple kinematics.

In the case of a well-sampled crossline MCG, a simple summation of these traces provides the predicted multiples at the apex of each event, as demonstrated in Figure 9. Next, an interpolation step is carried out over these sparse contributions to approximate the apex location of each event. Because of the low number of traces, a great deal of a priori knowledge should be put into this interpolation. Therefore, hyperbolic events are assumed, and a sparse inversion method describes the crossline MCGs with only a few apex-shifted hyperbolic events. This approach is adopted by Hokstad and Sollie (2003) and van Borselen et al. (2005). They modify the method to apex-shifted parabolic events, such that the expensive sparse transformation calculations can be carried out efficiently in the frequency domain with only minor loss in accuracy.

\section{On-the-fly interpolation}

An alternative to preprocessing the data to make them conform to the assumptions of the algorithm is to implement the algorithm assuming data are available wherever they are required. An on-the-fly interpolator is used to create the required traces from the recorded traces only when they are needed. These interpolated traces are then discarded after use.

This implementation philosophy allows the prediction algorithm to be made independent of the interpolator, in that it does not need to know anything about the actual, or regularized, acquisition geometries. The prediction algorithm is very simple and requires few parameters because it can expect to receive traces wherever they are required. This is accomplished without the need to create large, regularized data sets. Another reassuring feature of the algorithm is that it tends naturally to the ideal algorithm as the available data become increasingly well sampled.

With the advent of increasingly diverse acquisition geometries (multiazimuth, wide azimuth, rich azimuth, coil shooting, etc.), the flexibility of 3D SRME algorithms with respect to the geometry is becoming increasingly important. The detachment of the prediction algorithm from the interpolator means that the same prediction algorithm (and corresponding quality-control products) can be used regardless of acquisition geometry. The complexity is moved to the interpolation algorithm, which generally has its own associated quality-control products that can be used in conjunction with those for the prediction algorithm.

The main disadvantage of on-the-fly algorithms is that many interpolated traces are required. Typically, the aperture for a given target trace contains tens of thousands of DRPs, and each requires two interpolated traces. Therefore, only very simple interpolators are practical. Fortunately, however, because the MCGs contain so many convolutions, the quality of each interpolated trace can be quite low without this having a significant detrimental effect on the output when the MCG is stacked.

Probably the simplest class of interpolators that are applicable to this method are the nearest neighbor methods, which compute the in- terpolated trace from the nearest available trace (van Dedem and Verschuur, 2001; Kurin et al., 2006; Moore and Dragoset, 2008). There are many ways to define "nearest," but typically some weighted combination of the errors in midpoint, offset, and azimuth is used (Figure 10). These errors then provide quality-control information on the likely quality of the interpolated trace, and the errors can be accumulated in some way over the MCG to give an overall quality estimate for the predicted multiple. In practice, it is normal to try to correct the available trace for the errors in offset using a differentialmoveout correction in a manner similar to that used by Levin (2002) to simulate prestack data from a poststack volume. In principle, if a dip model is available, it is also possible to correct for errors in midpoint location.

Current 3D SRME implementations using nearest-neighbor, onthe-fly interpolation algorithms are considered state of the art for production processing, and several spectacular examples are shown later in the article. Although they can be very machine intensive, they are popular because the parameterization is simple, intuitive, and stable, and excellent results can be easily obtained by nonexpert users.

Algorithms based on regularization can also be run in an on-thefly mode, in that the regularized data need only be stored for as long as they are required. If the target traces are computed on a regular grid, then this can be made efficient because regularized shot and receiver gathers can be computed once and all of their contributions to the output model accumulated before discarding the regularized data. The ISR-DMO method is particularly amenable to this implementation.
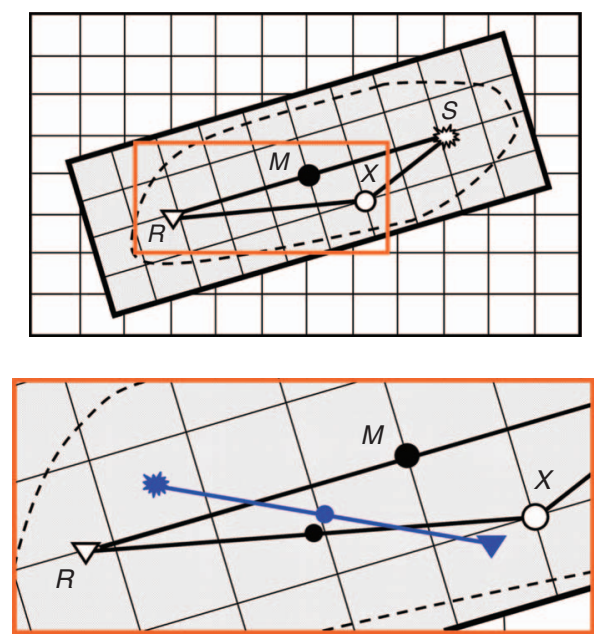

Figure 10. Plan view of an implementation of 3D SRME using onthe-fly interpolation. Surface multiples for trace $(S, R)$ are to be modeled. The dashed line illustrates the region that includes all downward reflection points for the multiples of interest, and the MCG aperture required to include these is drawn as a shaded box. The DRP grid is centered on the midpoint $M$ of trace $(S, R)$ and is aligned with its source-receiver line rather than with the background survey grid. An example DRP is annotated at $X$, which requires traces $(S, X)$ and $(X, R)$. The enlarged portion (bounded in orange) illustrates the selected nearest trace to $(X, R)$, using the selection criteria of minimum weighted errors in midpoint, offset, and azimuth. This selected trace is then modified on the fly to improve the correspondence to the desired trace $(X, R)$. 


\section{Approximations to 3D SRME}

The computational cost and complex input/output associated with any implementation of the full 3D SRME algorithm have naturally led to the development of many approximate algorithms. As always, the desire has been to reduce the cost of the algorithm yet maintain its accuracy.

This section focuses on cost reduction through algorithmic changes. The cost may also be reduced by other means, e.g., by choosing the aperture carefully (ultimately, in a space-variant manner) or by interpolating a sparse model of the predicted multiples, thereby reducing the number of target traces.

The simplest way to reduce the cost of 3D SRME is to reduce the number of convolutions required. Because the aperture and sampling are essentially fixed, this means finding a way to reuse convolutions for many target traces. Unfortunately, no convolutions are naturally duplicated from one target trace to the next, so it is desirable to be able to modify a convolution with a given geometry into that for a similar geometry. If the same modification applies to many convolutions in the MCG, then the modification can be performed after those convolutions are stacked, and the performance advantages are greatly enhanced. a)

b)
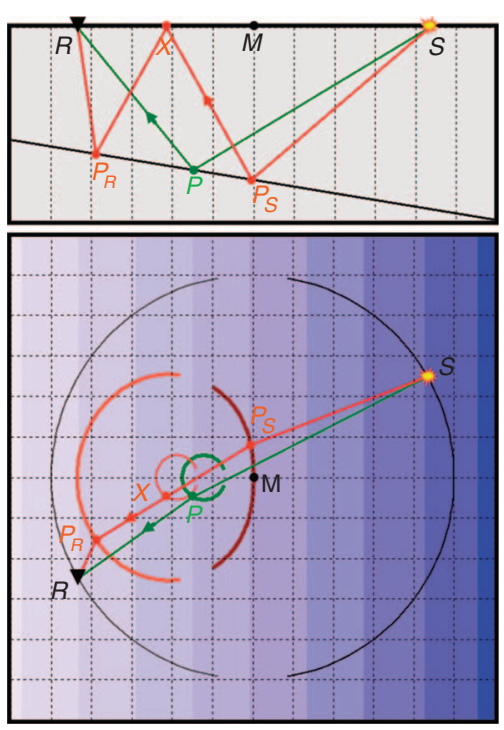

c)

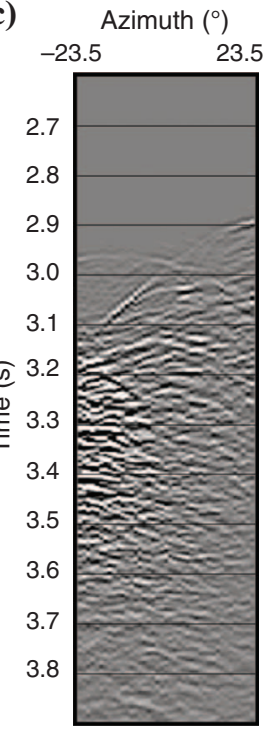

Figure 11. The effect of azimuth on primary and multiple reflections. (a) Side views of the primary (green) and multiple (red) raypaths for a planar water bottom with $10^{\circ}$ dip. The primary raypath extends from the shot $S$ to the receiver $R$ via an upward reflection at the water bottom $P$. The multiple raypath contains two upward reflection points $\left(P_{S}\right.$ and $\left.P_{R}\right)$, as well as the DRP, $X$. (b) A plan view of the same raypaths (arrowed green and red lines) with the same points annotated. The trace azimuth is $30^{\circ}$ to the dip direction. The arcs indicate the variation in the ray points as the azimuth is varied from $-80^{\circ}$ to $+80^{\circ}$ with the midpoint $M$ held constant. The primary reflection point $P$ is contained within a relatively small area of the water bottom as the azimuth is changed over a given range. In contrast, the multiple reflection points $P_{S}$ and $P_{R}$ traverse a large area for the same azimuth range and therefore see a greater variation in water depth and traveltime than the primary. (c) 3D-predicted multiples at 2500 $-m$ offset as a function of azimuth for a specific midpoint location in a field data set. Most multiples are very sensitive to azimuth, exhibiting up to $300 \mathrm{~ms}$ of timing variation (in either direction) over the $47^{\circ}$ azimuth range shown. (from Moore and Bisley, 2005)
Many workers have investigated these kinds of approaches. The $3 \mathrm{D}$ result in Figure 7 and the azimuth gather in Figure 11 were produced using one such algorithm. Despite the approximations that were made, this algorithm still allows the multiples to be predicted at true azimuth. The figures show that true-azimuth prediction was important in this case.

Although multiples are sensitive to azimuth in complex situations (Figure 11), there are simpler cases where the azimuth sensitivity can be neglected. If azimuth variations are small or 3D effects are weak, then predicting multiples at a fixed azimuth regardless of the actual azimuth of the target trace offers significant performance advantages while maintaining reasonable accuracy. This approach is generally applicable only to narrow-azimuth surveys, and the fixed azimuth (often termed zero azimuth) is the nominal inline azimuth of the survey. These algorithms are commonly termed zero-azimuth 3D SRME.

Of particular interest are algorithms that modify 2D SRME models into 3D SRME models (Matson and Abma, 2005). A 2D SRME model can be viewed as stacked MCGs in which the source, receiver, and DRP all have the same crossline location. If we restrict ourselves to zero-azimuth target traces, then we need to be able to modify these stacked, zero-crossline-offset convolutions into those that would have been computed if the DRP had some nonzero crossline offset from the source and receiver.

Figure 12 illustrates how this works. Suppose we want to predict multiples for a (zero-azimuth) target trace $\left(S_{t}, R_{t}\right)$ on a target line $L_{t}$. First, multiples are predicted independently for every line using a $2 D$ algorithm. The 2D-predicted multiples for line $L_{t}$ include contributions from DRPs such as $X_{t}$ that lie on the line, but they do not include contributions from DRPs such as $X_{t+2}$, which lies on line $L_{t+2}$. The contribution from this latter DRP, which ideally should be the convolution of the red traces $\left(S_{t}, X_{t+2}\right)$ and $\left(X_{t+2}, R_{t}\right)$, must be estimated from the convolutions, which are implicit in the 2D-predicted multiples. By making assumptions and approximations (Matson and Abma, 2005), we achieve this using operators that are kinematically similar to azimuthal moveout. The operators effectively transform zero-crossline-offset convolutions into their equivalents at finite crossline offset. However, such a correction requires a velocity mod$\mathrm{el}$; therefore, this class of approximate algorithm is somewhat re-

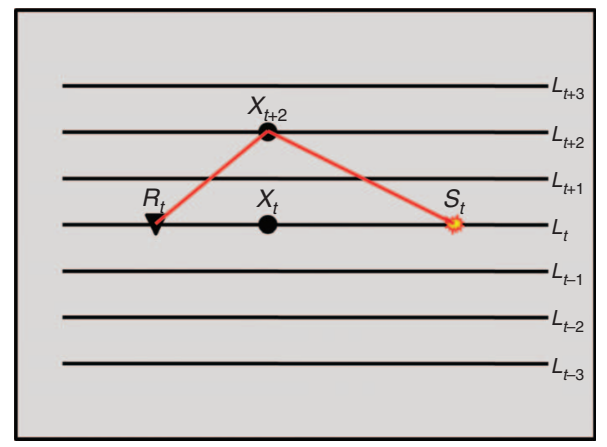

Figure 12. Schematic of zero-azimuth SRME. The target trace $\left(S_{t}, R_{t}\right)$ is on target line $L_{t}$. The 2D-predicted multiples for line $L_{t}$ include contributions from DRPs such as $X_{t}$ that lie on the line, but they do not include contributions from DRPs such as $X_{t+2}$, which lies on line $L_{t+2}$. The contribution from this latter DRP, which ideally should be the convolution of the red traces $\left(S_{t}, X_{t+2}\right)$ and $\left(X_{t+2}, R_{t}\right)$, must be estimated from the convolutions that are implicit in the $2 \mathrm{D}$ predicted multiples. 
moved from the purely data-driven ideal. Convolutions from a range of crossline offsets are then stacked to produce an estimate of the multiples that a 3D algorithm would predict.

\section{FIELD DATA EXAMPLES}

\section{Northwest Shelf, Australia}

Figure 13 compares 2D, zero-azimuth 3D, and true-azimuth 3D results for a field data set from the Northwest Shelf, Australia. A progressive improvement in the results is seen as the $2 \mathrm{D}$ assumption is first dropped, and then the zero-azimuth assumption is also removed. More details are given by Bisley and MacNeill (2008).

\section{Gulf of Mexico wide-azimuth data example}

In Figure 14, the difference between zero-azimuth and true-azimuth 3D multiple prediction is demonstrated for one shot record. The data are from a WAZ survey in the Keathley Canyon area. The shot record shown in Figure 14a is located at a 2.4-km nominal crossline offset. For more information on this data set, see Aaron et al. (2008) and Fromyr et al. (2008).

Figure $14 \mathrm{~b}$ shows the predicted multiples when the zero-azimuth assumption is used, meaning that the input data are first transformed

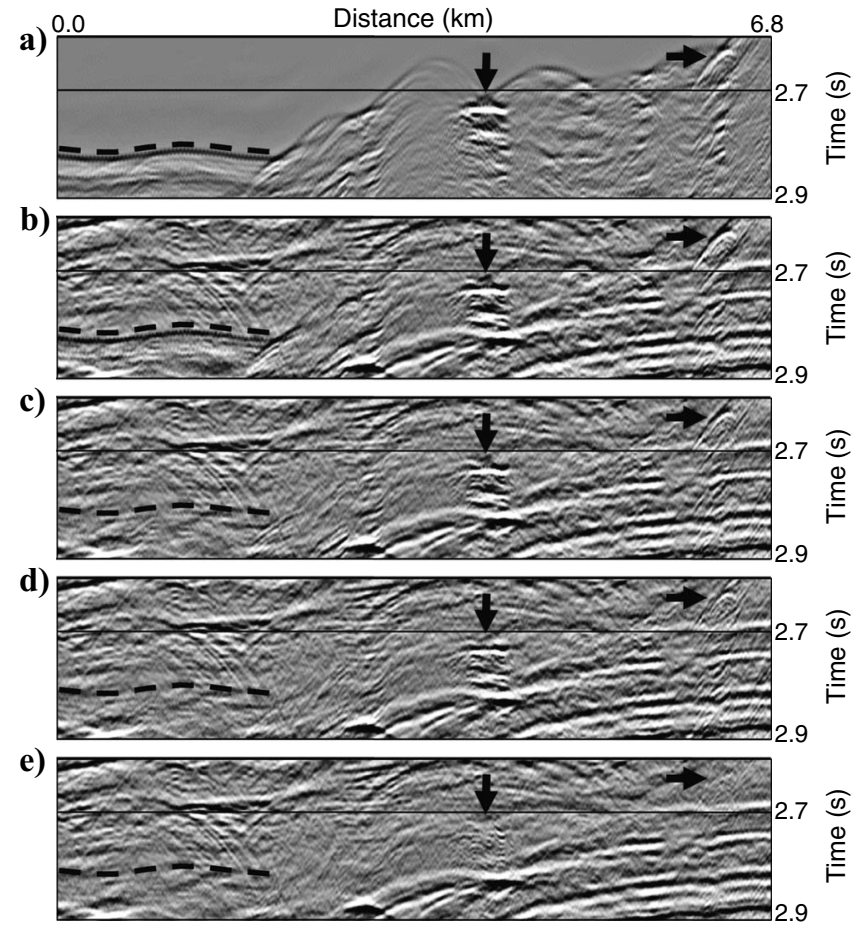

Figure 13. Field data example from the Northwest Shelf, Australia. (a) True-azimuth 3D SRME model. (b) Input. (c) The 2D SRME result. (d) Zero-azimuth 3D SRME result. (e) True-azimuth 3D SRME result. These panels display a progressive improvement after removing assumptions used in model generation. The model panel is included to help identify multiples in the other panels. The dashed line marks a simple region of water-bottom multiple that is well attenuated in all cases. The arrows to the right indicate areas of complex multiples. Some improvement over 2D SRME is seen when using zeroazimuth 3D SRME. A large improvement occurs when the zero-azimuth approximation is discarded. Displays are near-offset NMO stack panels from a sail subline. The match filtering parameterization is the same for all SRME results. to zero azimuth via an azimuth-moveout procedure, after which a zero-azimuth prediction takes place, according to Matson and Abma (2005). After that, predicted multiples can be projected to their original azimuths. For this complicated subsurface structure, in combination with the large crossline offset of the receiver location, the result is unacceptable errors in the prediction. Note that the events indicated with the red ovals are mispositioned with respect to the corresponding events in the input shot record and also do not have the proper lateral shape. The latter is especially visible for the upper area. In the lower red area, the relative amplitudes of the predicted multiples do not match the original ones. When the true-azimuth prediction method is used (Figure 14c), the results indicate predicted amplitudes, positioning, and shape of the multiples are in much better agreement with the multiples in the input data, as noted in the green areas.

\section{Diffracted-multiple example}

The final data example is from the full-azimuth circular-acquisition survey featured in Figure 1. The water-bottom reflection and several other reflectors just below it combine with a diffracting point to create a sequence of diffracted multiples (Figure 15a). A true-azimuth version of 3D SRME that includes nearest-neighbor, on-the-

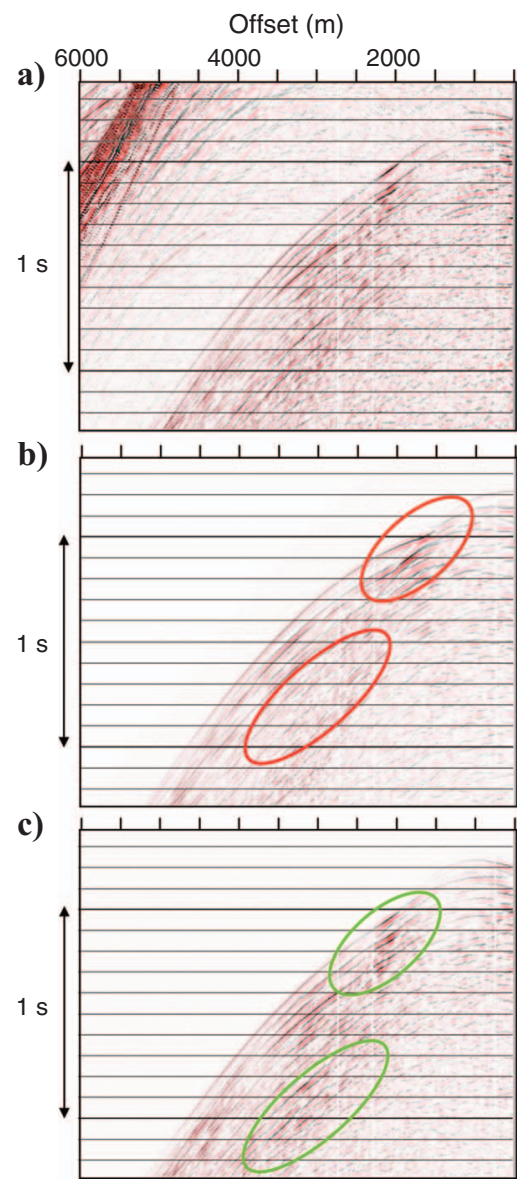

Figure 14. Comparison of (b) zero-azimuth 3D multiple prediction with (c) true-azimuth 3D multiple prediction for (a) one shot record extracted from a 3D survey in the Gulf of Mexico Keathley Canyon area. Note the errors in predicted multiples as observed in the zeroazimuth prediction (within the red ovals) are absent in the true-azimuth prediction (within the green ovals). (from Aaron et al., 2008) 
fly interpolation is applied to the data set (Figure 15b). The interpolation includes differential moveout (DNMO) to correct traces found by the search for discrepancies between their offsets and the offsets of the desired traces.

The DNMO correction used in this example is appropriate for primary reflections but not for diffractions. Why, then, is the attenuation of the multiple diffractions so successful? The answer to this question hearkens back to our discussion about errors in multiple contribution gathers. If those errors have no systematic bias, then they will stack out, leaving behind a good multiple prediction. Moore and Dragoset (2008) show a synthetic example of the timing errors in an MCG for a diffracted multiple; the errors are indeed unbiased.

\section{DISCUSSION}

Even a lengthy article such as this can only hope to give a general overview of the development of 3D SRME into the powerful process we know today. Although we have tried to cover the major aspects of that development, we acknowledge that it has been impossible to mention all of the research activity in this field. Furthermore, we have only been able to include, or reference, a very limited subset of the wide variety of case histories that have been published. We offer our apologies to those who have contributed to this field but have not received specific mention.

Despite recent advances, 3D SRME still has limitations. Adaptive subtraction remains a feature of the SRME workflow, though its job is becoming easier. We can expect our results to improve as a consequence of advances in adaptive subtraction technology, but the ultimate goal remains the refinement of the SRME multiple modelbuilding process to the point where adaptive subtraction is obviated.

Without a doubt, the main reason for imperfections in our 3D SRME models remains inadequate sampling of the wavefield. Future developments in acquisition technology will almost certainly improve this situation, but we remain a long way from the theoretical ideal. Advances in interpolation technology can also be expected to play their part in improving our models. In particular, we can expect the interpolation component of the on-the-fly methods to improve, especially as more computer power becomes available.

The theory and examples in this paper mirror those in the literature, in that there has been a focus on deepwater data. Shallow-water

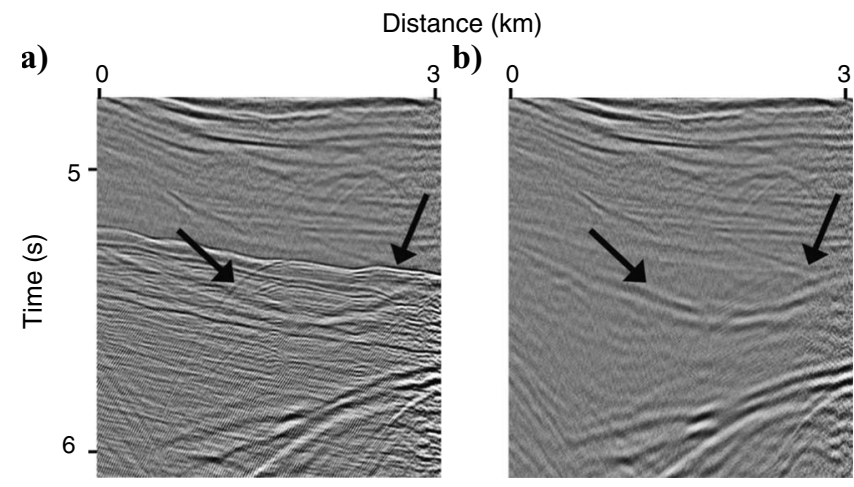

Figure 15. Stack section from the same survey as used in Figure 1. (a) Before multiple attenuation. The right arrow identifies the first-order water-bottom multiple. The left arrow identifies a group of several diffracted multiples. Each one arises from an upward reflection combined with an upgoing wave from the diffractor. (b) After application of on-the-fly interpolation, true-azimuth 3D SRME. data bring their own issues - most notably, the increased importance of near offsets and the need to iterate the prediction process to obtain correct amplitudes. Ideally, progress with respect to the first issue should be addressed with advances in acquisition technology, but there is also significant potential for new processing methods. An example is joint estimation of missing near-offset data and surface multiples (van Groenestijn and Verschuur, 2009).

Land data, of course, also suffer from surface multiples. The strong solid/air interface reflects almost all upgoing energy back into the subsurface, just as the water/air interface does in the marine case. However, in the land case, other issues often attract more attention. These include the attenuation of ground roll and other noise modes, and compensation for variations in near-surface conditions. Kelamis and Verschuur (2000) attempt to apply surface multiple removal to land data. They conclude that preprocessing is the key issue for applying data-driven methods to land data; any residual noise in the input data will be amplified in the predicted multiples, thus limiting the effectiveness of the subtraction. Workers de Maag et al. (2009) show that 3D SRME can remove long-period multiples from a wide-azimuth land data set. They attribute this success to the improved wavefield sampling of their WAZ acquisition. Although these two results are promising, further work and examples are needed before we can conclude that 3D SRME is, in general, as successful a process for land data as it is for marine data.

Finally, we should remember that the removal of multiples is largely motivated by our inability to process them properly; in fact, they contain useful information about the subsurface. Even though topics such as imaging or inverting multiples are well beyond the scope of this paper, we should not forget that, one day, articles such as this might become items of purely historical interest.

\section{CONCLUSIONS}

We have presented our perspective on the history, theory, implementation, and use of 3D surface-related multiple elimination. The theory itself is exact: With ideal field data and adequate computational resources, SRME multiple prediction should work perfectly, without assumptions or knowledge about the subsurface. In practice, field data are never ideal. Consequently, much of the research and development activity on 3D SRME has focused on determining how best to predict multiples for imperfect data. Many solutions have been proposed. The most accurate of these incorporate true-azimuth prediction, in which the algorithm honors the azimuths of the traces for which multiples are predicted. The currently favored approach to true-azimuth 3D SRME uses on-the-fly interpolation. In typical implementations of this method, some knowledge of the subsurface, such as moveout velocities, is required; this means that one no longer has a purely data-driven approach. The benefits of this pragmatism are great. Incorporating the data interpolation into the prediction decouples the entire SRME process from detailed dependence on survey geometry. Furthermore, as our data examples show, the results are excellent.

\section{ACKNOWLEDGMENTS}

We thank the Turkish Petroleum Company for granting permission to use Figures 1 and 15, Woodside Exploration Ltd. for Figure 13, and Petroleum Geo-Services for providing Figure 14. Jason Gardner of WesternGeco performed the 3D SRME processing for 
Figures 1 and 15, and Shuntay Fairley contributed to the figure preparation. I. Moore, R. Bisley, and B. Dragoset thank WesternGeco for allowing us the time to prepare this paper.

\section{APPENDIX A}

\section{AN INTUITIVE EXPLANATION OF SRME}

When SRME was introduced to the geophysical community, it met with a great deal of skepticism. Many geophysicists, being familiar with traditional methods of multiple attenuation, found it difficult to believe that all surface multiples could be predicted directly from surface recordings without specific knowledge of or assumptions about the subsurface. The short comeback to professed disbelief was that the properties of SRME follow directly from the mathematical theory of wave propagation. Although true, this reply was unsatisfactory to those not well versed in that theory. Thus, early proponents of SRME strove to explain the algorithm in ways that appealed to intuition rather than mathematical rigor. This appendix is based on those intuitive explanations.

Let's imagine the simple experiment shown in Figure A-1. A sound wave of arbitrary complexity is traveling through a nonabsorptive homogenous medium toward a listening post at point $R$. Between point $R$ and the sound wave, we choose some imaginary geometric surface such as $B$ and pose this question: If the sound wave is measured as it propagates through each point of surface $B$, do those measurements allow computation of the exact sound that will reach $R$ ? The answer to this question is yes, and the computational method is described approximately by the Huygens-Fresnel principle of wave propagation. ${ }^{5}$ The principle states that each point on measurement surface $B$ gives rise to a new pointlike source of sound that is equivalent to the sound incident at that point. In a homogeneous material, the sounds from these so-called secondary sources propagate as expanding spherical waves, and the total sound that reaches any subsequent point, such as $R$, is the superposition of those secondary

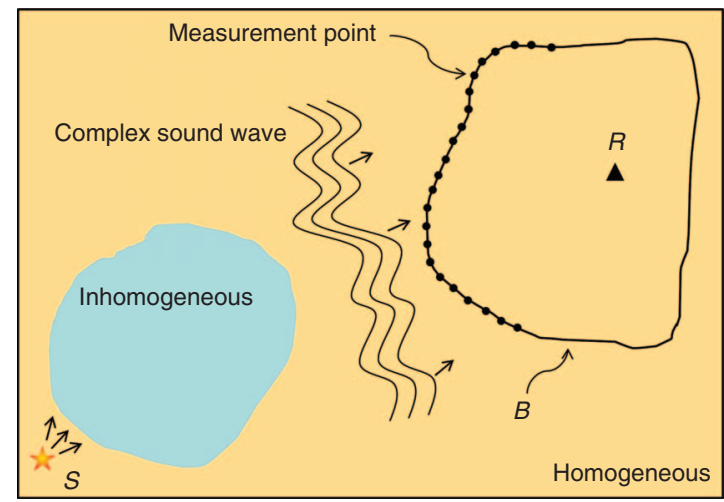

Figure A-1. Illustration of a wave propagation problem. A sound source $S$ and a sound detector $R$ are placed in a homogeneous material (yellow). Because of inhomogeneities (blue), the source creates a complex sound wave that propagates toward $R$. An arbitrary surface $B$ is defined so that it surrounds point $R$. The problem: If the sound wave incident on surface $B$ is measured, can that data be used to predict the sound that reaches $R$ ? (Note that $B$ is a 3D surface. It is shown here as a $2 \mathrm{D}$ surface to simplify the diagram.) sound waves (Figure A-2). Thus, given the measurements, the shape of surface $B$, and the location of point $R$ relative to surface $B$, the calculation of the sound that reaches $R$ is straightforward. A key property of the Huygens-Fresnel principle is that the nature of the original source of the sound at $S$ as well as the propagation phenomena the sound wavefield experiences prior to reaching surface $B$ is completely irrelevant to solving the posed problem.

Next, let's make the experiment more complicated. Suppose that the material inside of surface $B$ is absorptive and contains unknown inhomogeneities. In that case, the Huygens-Fresnel principle is still valid, but the calculation of the sound that reaches $R$ is no longer simple because the properties of the material now can cause all sorts of complicated phenomena to occur; that is, we no longer have simple spherical wave propagation to $R$ from the measurement points on $B$. Can we still calculate the sound at $R$ ? Again, the answer is yes, but now the experiment must include an additional set of measurements. As shown in Figure A-3, suppose that an impulsive sound source is activated at one of the measurement points on surface $B$ and the sound received at point $R$ is recorded. This procedure is repeated for every measurement point on $B$. In other words, the impulse response of the inhomogeneous material is measured between $R$ and every point on $B$. Analogous with filter theory, if a sound wave incident on a particular point of $B$ is measured, then its effect at point $R$ is given by the convolution of the measurement at $B$ with the appropriate impulse response. The total effect at $R$ of a sound wave incident on $B$ is thus a superposition of the convolutions between the measurements made at $B$ and the impulse responses recorded at $R$. The details of the material inside surface $B$ are completely irrelevant because their impact on sound-wave propagation has been fully characterized by the set of impulse response measurements at $R$.

Finally, in a sequence of steps, let's morph the second experiment into a configuration that resembles a seismic experiment (Figure A-4). First, surface B becomes a plane such that all sound that reaches $R$ must pass through it. Second, instead of being transparent, $B$ becomes a perfect reflector of sound. Although dramatic, this change

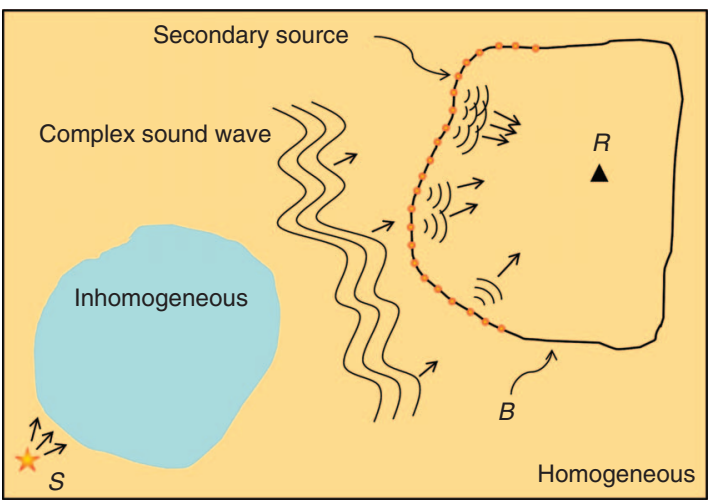

Figure A-2. An illustration of the Huygens-Fresnel principle of wave propagation. The answer to the question posed in Figure A- 1 is "Yes." According to the Huygens-Fresnel principle, the wavefield that propagates toward $R$ is a superposition of the wavefields from secondary sources placed on surface $B$. The figure shows a few of the secondary wavefields. The output of each secondary source is the sound incident on $B$ at that point. If there are no inhomogeneities within $B$, then the secondary wavefronts are spherical, which makes calculation of the sound that reaches $R$ easy.

\footnotetext{
${ }^{5} \mathrm{H}$. von Helmholtz and G. Kirchhoff found more mathematically rigorous solutions to this problem. Nevertheless, the Huygens-Fresnel principle provides an intuitive understanding of how the problem is solved.
} 
has no impact on the dynamics and kinematics of the experiment. Third, $B$ is identified as the sea surface, which is assumed to be a perfect reflector that changes the dynamics of the experiment by reversing the polarity of the reflections. Thus, the sound incident on $B$ (now the sea surface) is a $3 \mathrm{D}$ common-shot gather, the set of impulse response measurements from the second experiment is a 3D commonreceiver gather, and a stack of the trace-by-trace convolution of the two gathers predicts the surface multiples received at $R$ from a source firing at $S$. As in the imaginary experiment above, surface multiple prediction requires no details or assumptions about the sound propagation through the medium because all of that informa-

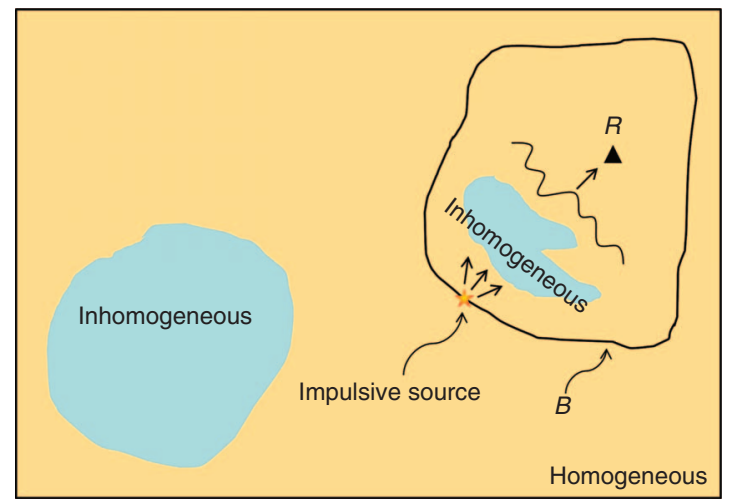

Figure A-3. A secondary experiment. When inhomogeneities are present within surface $B$, the wavefronts that propagate toward $R$ from the secondary sources are no longer spherical. If the inhomogeneities are unknown, then the wavefield at $R$ cannot be computed. This problem can be overcome by performing a second experiment in which the impulse responses at $R$ from each point on $B$ are measured. An example of one such measurement is shown here. A stack of convolutions of the incident wavefield at points on $B$ with the corresponding impulse responses predicts the sound received at $R$. a)

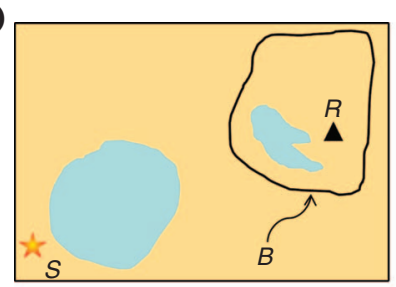

b)

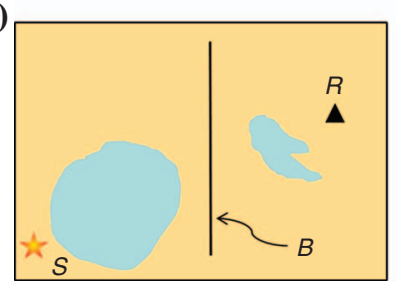

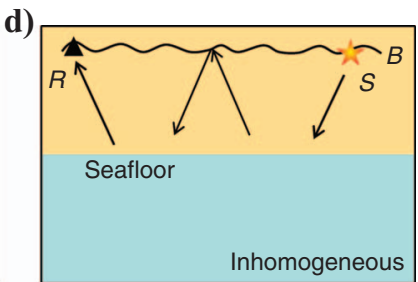

c)

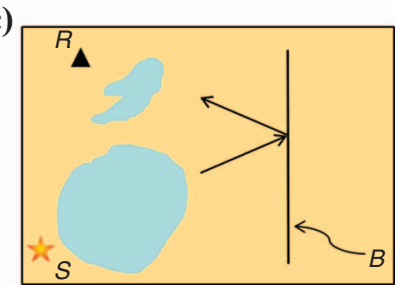

Figure A-4. Moving counterclockwise from the upper left, the experiment in Figure A-1 morphs into a seismic experiment. (a) The original experiment. (b) The measurement surface becomes a plane rather than an arbitrary shape. (c) A perfect reflector is positioned at the measurement plane. This alters neither the kinematics nor the dynamics of the experiment. (d) The measurement plane is now identified as the sea surface, the inhomogeneities become the seafloor and below, and the source and receiver are in the measurement plane. The wavefield from $S$ incident on $B$ is a common-shot gather, and the impulse responses at $R$ from points on $B$ are a common-receiver gather (with the source signature removed). tion is contained within the two sets of measurements.

Thinking about SRME as a sound propagation experiment, as the second one described above, makes many of its properties and requirements easy to understand. Clearly, every point in surface $B$ that receives sound from the source at $S$ should be considered a measurement point of the surface. This explains the aperture requirement for SRME as well as why holes in the aperture - from missing near offsets, for example - must somehow be filled. In addition, every measurement point on $B$ must have its impulse response measured at $R$, which explains why SRME ideally requires recording a shot at every measurement point on the surface. Also, a 3D common-receiver gather used in an SRME calculation is supposed to be a collection of impulse responses. If those responses are measured by a sound pulse that is not an impulse, then that sound pulse must be removed from the measurements. This is why SRME requires knowledge of the source signature. And if source signatures contain errors, then the predicted multiples will contain errors as well (see Figure 7 in Dragoset et al., 2006). Finally, when a 3D SRME calculation ignores trace azimuth, it's as if one performed the sound propagation experiment calculation by assuming the position of $R$ in Figure A-3 were somewhere other than its true position. Clearly, such a calculation would be faulty.

The experiment and data manipulation that we describe is a $3 \mathrm{D}$ version of the second term on the right in equation 10. Because that equation is in the frequency domain, the convolution of the data with themselves appears as a multiplication. The factor $A(\omega)=r_{0} S^{-1}(\omega)$ includes the reflection coefficient of the sea surface and a removal of the source signature.

\section{REFERENCES}

Aaron, P., R. O’Toole, S. Barnes, R. Hegge, and R. G. van Borselen, 2008, True-azimuth versus zero-azimuth 3-D multiple prediction in WATS processing: 78th Annual International Meeting, SEG, Expanded Abstracts, 2431-2435.

Abma, R., N. Kabir, K. H. Matson, S. Michell, S. A. Shaw, and B. McLain, 2005, Comparisons of adaptive subtraction methods for multiple attenuation: The Leading Edge, 24, 277-280.

Anstey, N. A., and P. Newman, 1966, The sectional auto-correlogram and the sectional retro-correlogram: Geophysical Prospecting, 14, 389-426.

Backus, M. M., 1959, Water reverberations - Their nature and elimination: Geophysics, 24, 233-261.

Baumstein, A., and M. T. Hadidi, 2006, 3D surface-related multiple elimination: Data reconstruction and application to field data: Geophysics, 71, no. 3, E25-E33.

Berkhout, A. J., 1982, Seismic migration, imaging of acoustic energy by wavefield extrapolation, Part A: Theoretical aspects: Elsevier Scientific Publ. Co., Inc.

Berkhout, A. J., and D. J. Verschuur, 1997, Estimation of multiple scattering by iterative inversion, Part I: Theoretical considerations: Geophysics, 62, 1586-1595.

Berryhill, J. R., and Y. C. Kim, 1986, Deep-water peg legs and multiple: Emulation and suppression: Geophysics, 51,2177-2184.

Biersteker, J., 2001, MAGIC: Shell's surface multiple attenuation technique: 71st Annual International Meeting, SEG, Expanded Abstracts, 1301-1304.

Bisley, R., and M. MacNeill, 2008, Application of true-azimuth 3D SRME in the Northwest Shelf, Australia: 78th Annual International Meeting, SEG, Expanded Abstracts, 2436-2440.

de Maag, J. W., A. J. Barhorst, T. M. van Dijk, F. Ernst, M. Klaassen, C. P. Matheny, P. Milcik, R. Romijn, M. van de Rijzen, J. L. Sheiman, A. P. E. Ten Kroode, H. van Voorst Vader, K. de Vos, D. Wright, and P. M. Zwartjes, 2009, 3D WAZ land acquisition driving new processing developments: 71st Conference \& Technical Exhibition, EAGE, Extended Abstracts, V012.

Dix, C. H., 1948, The existence of multiple reflections: Geophysics, 13, 49-50.

Dragoset, W. H., 1993, Surface multiple attenuation - Theory, practical issues, examples: 55th Conference \& Technical Exhibition, EAGE, Extended Abstracts, B027. 
Dragoset, W. H., and Ž. Jeričević, 1998, Some remarks on surface multiple attenuation: Geophysics, 63, 772-789.

Dragoset, W. H., and S. MacKay, 1993, Surface multiple attenuation and subsalt imaging: 63rd Annual International Meeting, SEG, Expanded Abstracts, 1099-1102.

Dragoset, B., I. Moore, and C. Kostov, 2006, The impact of field-survey characteristics on surface-related multiple attenuation: Geophysical Prospecting, 54, 781-791.

Fokkema, J. T., and P. M. van den Berg, 1990, Removal of surface-related phenomena: The marine case: 60th Annual International Meeting, SEG, Expanded Abstracts, 1689-1692.

Fromyr, E., C. James, and P. E. Dhelie, 2008, Crystal — A wide azimuth towed-streamer program: Acquisition design analysis: 70th Conference \& Technical Exhibition, EAGE, Extended Abstracts, G010.

Hampson, D., 1986, Inverse velocity stacking for multiple elimination: Journal of the Canadian Society of Exploration Geophysics, 22, 44-55.

Hokstad, K., and R. Sollie, 2003, 3D surface-related multiple elimination using parabolic sparse inversion: 73rd Annual International Meeting, SEG, Expanded Abstracts, 1961-1964.

Jakubowicz, H., 1998, Wave equation prediction and removal of interbed multiples: 68th Annual International Meeting, SEG, Expanded Abstracts, 1527-1530.

Kabir, M. M. N., and D. J. Verschuur, 1995, Restoration of missing offsets by parabolic Radon transform: Geophysical Prospecting, 43, 347-368.

Kelamis, P. G., and D. J. Verschuur, 2000, Surface-related multiple elimination on land seismic data - Strategies via case studies: Geophysics, 65, 719-734.

Kennett, B. L. N., 1979a, Theoretical reflection seismograms for elastic media: Geophysical Prospecting, 27, 301-321.

, 1979b, The suppression of surface multiples on seismic records: Geophysical Prospecting, 27, 584-600.

Kleemeyer, G., S. E. Pettersson, R. Eppenga, C. J. Haneveld, J. Biersteker, and R. den Ouden, 2003, It's Magic: Industry first 3D surface multiple elimination and pre-stack depth migration on Ormen Lange: 65 th Conference \& Technical Exhibition, EAGE, Extended Abstracts, B043.

Kurin, E., M. S. Denisov, and D. Lokshtanov, 2006, A method for 3D surface-related multiples prediction in case of coarse sampling: 68th Conference \& Technical Exhibition, EAGE, Extended Abstracts, B032.

Levin, S. A., 2002, Prestack poststack 3D multiple prediction: 72nd Annual International Meeting, SEG, Expanded Abstracts, 2110-2113.

Lin, D., J. Young, Y. Huang, and M. Hartmann, 2004, 3D SRME application in the Gulf of Mexico: A case study: 74th Annual International Meeting, SEG, Expanded Abstracts, 1257-1260.

Lin, D., J. Young, W. Lin, M. Griffiths, and M. Hartmann, 2005, 3D SRME practice for better imaging: 67th Conference \& Technical Exhibition, EAGE, Extended Abstracts, A030.

Matson, K. H., and R. Abma, 2005, Fast 3D surface-related multiple elimination using azimuth moveout for multiples: 75th Annual International Meeting, SEG, Expanded Abstracts, 2064-2067.

Matson, K., and D. Corrigan, 2000, A 2.5D method for attenuating free surface multiples based on inverse scattering series: Offshore Technology Conference, doi:.

Matson, K. H., S. Michell, R. Abma, E. Shoshitaishvili, M. C. Williams, I. Ahmed, J. D. Oldroyd, and R. R. Fisher, 2004, Advanced subsalt imaging and 3D surface multiple attenuation in Atlantis: A case study: 74th Annual International Meeting, SEG, Expanded Abstracts, 1269-1272.

Mayne, W. H., 1962, Common reflection point horizontal data stacking techniques: Geophysics, 27, 927-938.

McHugo, S., A. Cooke, and P. Charron, 2009, 3D Surface related multiple elimination in the presence of a complex water bottom geometry - A case study from offshore Nigeria: 79th Annual International Meeting, SEG, Expanded Abstracts, 3068-3072

Moldoveanu, N., J. Kapoor, and M. Egan, 2008, A single-vessel method for wide-azimuth towed-streamer acquisition: 78th Annual International
Meeting, SEG, Expanded Abstracts, 65-69.

Moore, I., and R. Bisley, 2005, 3D surface-related multiple prediction (SMP): A case history: The Leading Edge, 24, 270-274.

Moore, I., and B. Dragoset, 2008, General surface multiple prediction: A flexible 3D SRME algorithm: First Break, 26, September, 89-100.

Nekut, A. G., 1998, 3D surface-related multiple prediction: 68th Annual International Meeting, SEG, Expanded Abstracts, 1511-1514.

Pica, A., G. Poulain, B. David, M. Magesan, S. Baldock, T. Weisser, P. Hugonnet, and P. Herrmann, 2005, 3D surface-related multiple modeling: The Leading Edge, 24, 292-296.

Riley, D. C., and J. F. Claerbout, 1976, 2-D multiple reflections: Geophysics, 41, 592-620.

Ross, W. S., Y. Yu, and F. A. Gasparotto, 1999, Traveltime prediction and suppression of 3-D multiples: Geophysics, 64, 261-277.

Schneider, W. A., 1978, Integral formulation for migration in two and three dimensions: Geophysics, 43, 49-76.

Sen, M. K., F. Liu, P. L. Stoffa, and J. T. Fokkema, 1998, A unified treatment of free surface multiple elimination algorithms: Journal of Seismic Exploration, 7, 129-143.

van Borselen, R., G. Fookes, and J. Brittan, 2003, Target-oriented adaptive subtraction in data-driven multiple removal: The Leading Edge, 22, 340-343.

van Borselen, R., M. A. Schonewille, and R. F. Hegge, 2005, 3D surface-related multiple elimination: Acquisition and processing solutions: The Leading Edge, 24, 260-268.

van Dedem, E. J., and D. J. Verschuur, 1997, Analysis of surface-related multiples in 3D media: 67th Annual International Meeting, SEG, Expanded Abstracts, 1180-1183.

- 2000, 3D surface-related multiple prediction - An inversion approach: 70th Annual International Meeting, SEG, Expanded Abstracts, 1965-1968.

$-2001,3 \mathrm{D}$ surface multiple prediction using sparse inversion: 71st Annual International Meeting, SEG, Expanded Abstracts, 1285-1288.

$-, 2005,3 \mathrm{D}$ surface-related multiple prediction — A sparse inversion approach: Geophysics, 70, no. 3, V31-V43.

van Groenestijn, G. J. A., and D. J. Verschuur, 2009, Estimating primaries by sparse inversion and application to near-offset data reconstruction: Geophysics, 74, no. 3, A23-A28.

Verschuur, D. J., 1991, Surface-related multiple elimination, an inversion approach: Ph.D. dissertation, Delft University of Technology.

- 1992, Surface-related multiple elimination in terms of Huygens' sources: Journal of Seismic Exploration, 1, 49-59.

, 2006, Seismic multiple removal techniques, past, present and future: EAGE.

Verschuur, D. J., and A. J. Berkhout, 1997, Estimation of multiple scattering by iterative inversion, Part II: Practical aspects and examples: Geophysics, 62, 1596-1611.

Verschuur, D. J., A. J. Berkhout, and C. P. A. Wapenaar, 1988, Elimination of surface-related multiply reflected and converted events: 58th Annual International Meeting, SEG, Expanded Abstracts, 1017-1020.

- 1992, Adaptive surface-related multiple elimination: Geophysics, 57, 1166-1177.

Wapenaar, C. P. A., and A. J. Berkhout, 1989, Elastic wave field extrapolation: Redatuming of single- and multi-component seismic data: Elsevier Scientific Publ. Co., Inc.

Watson, R. J., 1965, Decomposition and suppression of multiple reflections: Geophysics, 30, 54-71.

Weglein, A. B., F. A. F. Gasparotto, P. M. Carvalho, and R. H. Stolt, 1997, An inverse-scattering series method for attenuating multiples in seismic reflection data: Geophysics, 62, 1975-1989.

Wiggins, J. W., 1988, Attenuation of complex water-bottom multiples by wave-equation-based prediction and subtraction: Geophysics, 53, $1527-1539$ 